\title{
Decidable Logics Combining Heap Structures and Data
}

\author{
P. Madhusudan \\ University of Illinois at \\ Urbana-Champaign, USA \\ madhu@illinois.edu
}

\author{
Gennaro Parlato \\ LIAFA, CNRS and University of Paris \\ Diderot, France \\ gennaro@liafa.jussieu.fr
}

\author{
Xiaokang Qiu \\ University of Illinois at \\ Urbana-Champaign, USA \\ qiu2@illinois.edu
}

\begin{abstract}
We define a new logic, STRAND, that allows reasoning with heapmanipulating programs using deductive verification and SMT solvers. STRAND logic ("STRucture ANd Data" logic) formulas express constraints involving heap structures and the data they contain; they are defined over a class of pointer-structures $\mathcal{R}$ defined using MSO-defined relations over trees, and are of the form $\exists \vec{x} \forall \vec{y} \varphi(\vec{x}, \vec{y})$, where $\varphi$ is a monadic second-order logic (MSO) formula with additional quantification that combines structural constraints as well as data-constraints, but where the data-constraints are only allowed to refer to $\vec{x}$ and $\vec{y}$.

The salient aspects of the logic are: (a) the logic is powerful, allowing existential and universal quantification over the nodes, and complex combinations of data and structural constraints; (b) checking Hoare-triples for linear blocks of statements with preconditions and post-conditions expressed as Boolean combinations of existential and universal STRAND formulas reduces to satisfiability of a STRAND formula; (c) there are powerful decidable fragments of STRAND, one semantically defined and one syntactically defined, where the decision procedure works by combining the theory of MSO over trees and the quantifier-free theory of the underlying data-logic. We demonstrate the effectiveness and practicality of the logic by checking verification conditions generated in proving properties of several heap-manipulating programs, using a tool that combines an MSO decision procedure over trees (MONA) with an SMT solver for integer constraints (Z3).
\end{abstract}

Categories and Subject Descriptors F.3.1 [Logics and Meanings of Programs]: Specifying and Verifying and Reasoning about Programs: Mechanical Verification; D.2.4 [Software Engineering]: Software/Program Verification: Assertion checkers; F.1.1 [Theory of Computation]: Models of Computation: Automata

General Terms Algorithms, Reliability, Theory, Verification

Keywords heap analysis, SMT solvers, monadic second-order logic, combining decision procedures, automata, decidability

\section{Introduction}

A fundamental component of analysis techniques for complex programs is logical reasoning. The advent of efficient SMT solvers (satisfiability modulo theory solvers) have significantly advanced

Permission to make digital or hard copies of all or part of this work for personal or classroom use is granted without fee provided that copies are not made or distributed for profit or commercial advantage and that copies bear this notice and the full citation on the first page. To copy otherwise, to republish, to post on servers or to redistribute to lists, requires prior specific permission and/or a fee.

POPL'11, January 26-28, 2011, Austin, Texas, USA

Copyright (C) 2011 ACM 978-1-4503-0490-0/11/01 .. \$10.00 the techniques for the analysis of programs. SMT solvers check satisfiability in particular theories (e.g. integers, arrays, theory of uninterpreted functions, etc.), and are often restricted to quantifierfree fragments of first-order logic, but support completely automated and efficient decision procedures for satisfiability. Moreover, by using techniques that combine theories, larger decidable theories can be obtained. The Nelson-Oppen framework [22] allows generic combinations of quantifier-free theories, and has been used in efficient implementations of combinations of theories using a SAT solver that queries decision procedures of component theories.

Satisfiability solvers for theories are tools that advance several analysis techniques. They are useful in test-input generation, where the solver is asked whether there exists an input to a program that will drive it along a particular path; see for example [12]. SMT solvers are also useful in static-analysis based on abstract interpretation, where the solver is asked to compute precise abstract transitions (for example see SLAM [2] for predicate abstraction and TVLA $[17,27]$ for shape-analysis). Solvers are also useful in classical deductive verification, where Hoare-triples that state preconditions and post-conditions can be transformed into verification conditions whose validity is checked by the solver; for example BoogIE [3] and ESC/Java [11] use SMT solvers to prove verification conditions.

One of the least understood classes of theories, however, are theories that combine heap-structures and the data they contain. Analysis of programs that manipulate dynamically allocated memory and perform destructive pointer-updates while maintaining datastructure invariants (like a binary search tree), requires reasoning with heaps with an unbounded number of nodes with data stored in them. Reasoning with heap structures and data poses fundamental challenges due to the unboundedness of the data-structures. First, for a logic to be useful, it must be able to place constraints on all parts of the structure (e.g. to say a list is sorted), and hence some form of universal quantification over the heap is absolutely necessary. This immediately rules out classical combinations of theories, like the Nelson-Oppen scheme [22], which caters only to quantifierfree theories. Intuitively, given a constraint on heap structures and data, there may be an infinite number of heaps that satisfy the structural constraints, and checking whether any of these heaps can be extended with data to satisfy the constraint cannot be stated over the data-logic (even if it has quantification).

There have been a few breakthroughs in combining heap structures and data recently. For instance, HAVOC [16] supports a logic that ensures decidability using a highly restrictive syntax, and CSL [7] extends the HAVOC logic mechanism to handle constraints on sizes of structures. However, both these logics have very awkward syntax that involve the domain being partially ordered with respect to sorts, and the logics are heavily curtailed so that the decision procedure can move down the sorted structures hierarchically and hence terminate. Moreover, these logics cannot express even simple properties on trees of unbounded depth, like the property 
that a tree is a binary search tree. More importantly, the technique for deciding the logic is encoded in the syntax, which in turn narrowly aims for a fast reduction to the underlying data-logic, making it hard to extend or generalize.

In this paper, we propose a new fundamental technique for deciding theories that combine heap structures and data, for fragments of a logic called STRAND.

The logic STRAND: We define a new logic called STRAND (for "STRucture ANd Data"), that combines a powerful heap-logic with an arbitrary data-logic. STRAND formulas are interpreted over a class of data-structures $\mathcal{R}$, and are of the form $\exists \vec{x} \forall \vec{y} \varphi(\vec{x}, \vec{y})$, where $\varphi$ is a formula that combines a complete monadic second-order logic over the heap-structure (and can have additional quantification), and a data-logic that can constrain the data-fields of the nodes referred to by $\vec{x}$ and $\vec{y}$.

The heap-logic in STRAND is derived from the rich logic tradition of designing decidable monadic second-order logics over graphs, and is extremely expressive in defining structural shapes and invariants. STRAND formulas are interpreted over a recursively defined class of data-structures $\mathcal{R}$, which is defined using a regular set of skeleton trees with MSO-defined edge-relations (pointerrelations) between them. This way of recursively defining datastructures is not new, and was pioneered by the PALE system [20], which reasons with purely structural properties of heaps defined in a similar manner. In fact, the notion of graph types [14] is a convenient and simple way to define data-structure types and invariants, and is easily expressible in our scheme. Data-structures defined over skeleton trees have enough expressive power to state most data-structure invariants of recursively defined data-structures, including nested lists, threaded trees, cyclic and doubly-linked lists, and separate or loosely connected combinations of these structures. Moreover, they present a class of graphs that have a decidable MSO theory, as MSO on these graphs can be interpreted using MSO over trees, which is decidable. In fact, graphs defined this way are one of the largest classes of graphs that have a decidable MSO theory.

As we show in this paper, the STRAND logic is well-suited to reasoning with programs. In particular, assume we are given a (straight-line) program $P$, a pre-condition on the data-structure expressed as a set of recursive structures $\mathcal{R}$, and a pre-condition and a post-condition expressed in a sub-fragment of STRAND that allows Boolean combinations of the existential and universal fragments. We show that checking the invalidity of the associated Hoare-triple reduces to the satisfiability problem of STRAND over a new class of recursive structures $\mathcal{R}_{P}$.

Note that despite its relative expressiveness in allowing quantification over nodes, STRAND formulas cannot express certain constraints such as those that constrain the length of a list of nodes (e.g., to express that the number of black nodes on all paths in a red-black tree are the same), nor express the multi-set of datavalues stored in a data-structure (e.g., to express that one list's data contents are the same as that of another list). We hope that future work will extend the results in this paper to handle such constraints.

Decidable fragments of STRAND: The primary contribution of this paper is in identifying decidable fragments of STRAND. We define two such fragments, one which is a semantic fragment $\mathrm{STRAND}_{\text {dec }}^{\text {sem }}$ that defines the largest class that can exploit our combination mechanism for decidability, and the other a smaller but syntactic fragment $\mathrm{STRAND}_{\text {dec }}$.

The decision procedures work through a notion called satisfiability-preserving embeddings. Intuitively, for two heap structures (without data) $S$ and $S^{\prime}, S$ satisfiability-preservingly embeds in $S^{\prime}$ with respect to a STRAND formula $\psi$ if there is an embedding of the nodes of $S$ in $S^{\prime}$ such that no matter how the data-logic constraints are interpreted, if $S^{\prime}$ satisfies $\psi$, then so will the submodel $S$ satisfy $\psi$, by inheriting the data-values. We define the notion of satisfiability-preserving embeddings so that it is entirely structural in nature, and is definable using MSO on an underlying graph that simultaneously represents $S, S^{\prime}$, and the embedding of $S$ in $S^{\prime}$.

If $S$ satisfiability-preservingly embeds in $S^{\prime}$, then clearly, when checking for satisfiability, we can ignore $S^{\prime}$ if we check satisfiability for $S$. More generally, the satisfiability check can be done only for the minimal structures with respect to the partial-order (and well-order) defined by satisfiability-preserving embeddings.

The semantic decidable fragment STRAND the class of all formulas for which the set of minimal structures with respect to satisfiability-preserving embeddings is finite, and where the quantifier-free theory of the underlying data-logic is decidable. Though this fragment of STRAND is semantically defined, we show that it is syntactically checkable. Given a STRAND formula $\psi$, we show that we can build a regular finite representation of all the minimal models with respect to satisfiability-preserving embeddings, even if it is an infinite set, using automata-theory. Then, checking whether the number of minimal models is finite is decidable. If the set of minimal models is finite, we show how to enumerate the models, and reduce the problem of checking whether they admit a data-extension that satisfies $\psi$ to a formula in the quantifier-free fragment of the underlying data-logic, which can then be decided.

We also define a syntactic decidable fragment of STRAND, STRAND $_{d e c}$, which is a subfragment of the semantic class STRAND Sec $^{\text {sem }}$. In this fragment, we distinguish two kinds of binary relations in the heap, elastic and non-elastic relations. Intuitively, a relation is elastic if for every model $M$ and submodel $M^{\prime}$, the relation holds on a pair of nodes of $M^{\prime}$ iff the relation holds for the corresponding pair of nodes in $M$. Given a relation $R$, we show it is also decidable whether $R$ is an elastic relation. $\mathrm{STRAND}_{d e c}$ formulas are then of the form $\exists \vec{x} \forall \vec{y} \varphi(\vec{x}, \vec{y})$, where (a) $\varphi$ has no additional quantification, and (b) the atomic non-elastic structural relations in $\varphi$ compare only variables in $\vec{x}$. We show that $\mathrm{STRAND}_{d e c}$ formulas always have a finite number of minimal models with respect to satisfiability-preserving embeddings, and are hence decidable using the decision procedure for the semantic fragment STRAND dec $^{\text {sem }}$.

We report also on an implementation of the above decision procedures. For the structural phase, we use MONA [13], a powerful tool for deciding MSO over trees which, despite its theoretical non-elementary worst-case complexity, works very efficiently on realistic examples, by combining a variety of techniques including tree-automata minimization, BDDs, and guided tree automata. The quantifier-free data-logic we use is the quantifier-free logic of linear arithmetic, and we use the SMT solver Z3 to handle these constraints. We have proved several heap-manipulating programs correct including programs that search and insert into sorted lists, reverse sorted lists, and perform search, insertion, and rotation on binary-search trees.

In each of these cases, the verification conditions were always expressible in the syntactic fragment $\mathrm{STRAND}_{d e c}$, and hence in the semantic decidable fragment STRAND dec $^{\text {sem }}$, supporting our thesis that the decidable fragment is natural and useful.

In summary, we present a general decidability technique for combining heap structures and data, identify semantically a decidable fragment $\mathrm{STRAND}_{d e c}^{\text {sem }}$, demonstrate a syntactically-defined subfragment STRAND dec, and present experimental evaluation to show that the decidable combination is expressive and efficiently solvable. We believe that this work breaks new ground in combining heap structures and data, and the technique may also pave the way for defining decidable fragments of other logics, such as separation logic, that combine structures and data.

\section{Motivating examples and logic design}

The goal of this section is to present an overview of the issues involved in finding decidable logics that combine heap structure 
and data, which sets the stage for defining the decidable fragments of the logic STRAND, and motivates the choices in our logic design using simple examples on lists.

Let us consider lists in this section, where each node $u$ has a data-field $d(u)$ that can hold a value (say an integer), and with two variables head and tail pointing to the first and last nodes of the list, respectively. Consider first-order logic, where we are allowed to quantify over the nodes of the list, and further, for any node $x$, allowed to refer to the data-field of $x$ using the term $d(x)$. Let $x \rightarrow y$ denote that $y$ is the successor of $x$ in the list, and let $x \rightarrow^{*} y$ denote that $x$ is the same as $y$ or precedes $y$ in the list.

EXAMPLE 2.1. Consider the formula:

$$
\begin{gathered}
\varphi_{1}: \quad d(\text { head })=c_{1} \wedge d(\text { tail })=c_{2} \wedge \\
\forall y_{1} \forall y_{2}\left(\left(y_{1} \rightarrow^{*} y_{2}\right) \Rightarrow d\left(y_{1}\right) \leq d\left(y_{2}\right)\right)
\end{gathered}
$$

The above says that the list must be sorted and that the head of the list must have value $c_{1}$ and the tail must have value $c_{2}$. Note that the formula is satisfiable iff $c_{1} \leq c_{2}$, and in which case it is actually satisfied by a list containing just two elements, pointed to by head and tail, with values $c_{1}$ and $c_{2}$, respectively.

In fact, the property that the formula is satisfiable by a twoelement list has nothing really to do with the data-constraints involved in the above formula. Assume that we have no idea as to what the data-constraints mean, and hence look upon the above formula by replacing all the data-constraints using uninterpreted predicates $p_{1}, p_{2}, \ldots$ to get the formula:

$$
\begin{aligned}
\widehat{\varphi}_{1}: \quad p_{1}(d(\text { head })) \wedge p_{2}(d(\text { tail })) \wedge \\
\forall y_{1} \forall y_{2}\left(\left(y_{1} \rightarrow^{*} y_{2}\right) \Rightarrow p_{3}\left(d\left(y_{1}\right), d\left(y_{2}\right)\right)\right)
\end{aligned}
$$

Now, we do not know whether the formula is satisfiable (for example, $p_{1}$ may be unsatisfiable). But we still do know that two-element lists are always sufficient. In other words, if there is a list that satisfies the above formula, then there is a two-element list that satisfies $i t$. The argument is simple: take any list $l$ that satisfies the formula, and form a new list $l^{\prime}$ that has only the head and tail of the list $l$, with an edge from head to tail, and with data values inherited from $l$ (see figure below). It is easy to see that $l^{\prime}$ satisfies the formula as well, since whenever two nodes are related by $\rightarrow^{*}$ in the list $l^{\prime}$, the corresponding elements in $l$ are similarly related. This property,

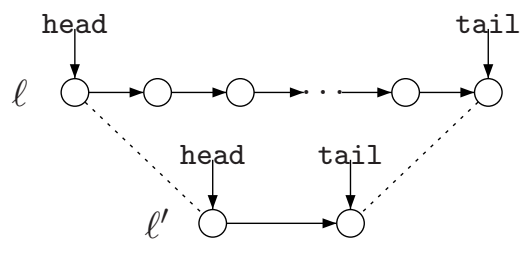

of course, does not hold on all formulas, as we see in the example below.

\section{EXAMPLE 2.2. Consider the formula:}

$$
\begin{gathered}
\varphi_{2}: \quad d(\text { head })=c_{1} \wedge d(\text { tail })=c_{2} \wedge \\
\forall y_{1} \forall y_{2}\left(\left(y_{1} \rightarrow y_{2}\right) \Rightarrow d\left(y_{2}\right)=d\left(y_{1}\right)+1\right)
\end{gathered}
$$

The above says that the values in the list increase by one as we go one element down the list, and that the head and tail of the list have values $c_{1}$ and $c_{2}$, respectively. This formula is satisfiable iff $c_{1}<c_{2}$. However, there is no bound on the size of the minimal model that is independent of the data-constraints. For example, if $c_{1}=1$ and $c_{2}=10^{6}$, then the smallest list that satisfies the formula has a million nodes. In other words, the data-constraints place arbitrary lower bounds on the size of the minimal structure that satisfies the formula.

Intuitively, the formula $\varphi_{2}$ refers to successive elements in the list, and hence a large model that satisfies the formula is not neces- sarily contractible to a smaller model. The formula $\varphi_{1}$ in the sortedness example (Example 2.1) refers to pairs of elements that were reachable, leading to contraction of large models to small ones.

Recall that the design principle of the decidable fragment of STRAND is to examine the structural constraints in a formula $\varphi$, and enumerate a finite set of structures such that the formula is satisfiable iff it one of these structures can be populated with values to satisfy the formula. This strategy necessarily fails for the above formula $\varphi_{2}$, as there is no class of finite structures that adequately captures all models of the formula, independent of the data-constraints. The sortedness formula $\varphi_{1}$ in the first example is part of the decidable fragment of STRAND, while $\varphi_{2}$ is outside of it.

EXAMPLE 2.3. Consider the formula:

$$
\begin{aligned}
& \varphi_{3}: d(\text { head })=c_{1} \wedge d(\text { tail })=c_{2} \wedge \\
& \forall y_{1}\left(\left(y_{1} \neq \text { tail }\right) \Rightarrow \exists y_{2}\left(d\left(y_{2}\right)=d\left(y_{1}\right)+1\right)\right)
\end{aligned}
$$

This formula says that for any node $n$ except the tail, there is some node $n^{\prime}$ that has the value $d(n)+1$. Notice that the formula is satisfiable if $c_{1}<c_{2}$, but still there is no a priori bound on the minimal model that is independent of the data-constraints. In particular, if $c_{1}=0$ and $c_{2}=10^{6}$, then the smallest model is a list with $10^{6}$ nodes. Moreover, the reason why the bounded structure property fails is not because of the data-constraints referring to successive elements as in Example 2.2, but rather because the above formula has a $\forall \exists$ prefix quantification of data-variables. Formulas where an existential quantification follows a universal quantification in the prefix seldom have bounded models, and STRAND hence only allows formulas with $\exists^{*} \forall^{*}$ quantification prefixes. Note that quantification of structure variables (variables that quantify over nodes but whose data-field is not referenced in the formula) can be arbitrary, and in fact we allow STRAND formulas to even have set quantifications over nodes.

The Bernays-Schönfinkel-Ramsey class: Having motivated formulas with the $\exists^{*} \forall^{*}$ quantification, it is worthwhile to examine this fragment in classical first-order logic (over arbitrary infinite universes), which is known as the Bernays-Schönfinkel-Ramsey class, and is a classical decidable fragment of first-order logic [6].

Consider first a purely relational vocabulary (assume there are no functions and even no constants). Then, given a formula $\exists \vec{x} \forall \vec{y} \varphi(\vec{x}, \vec{y})$, let $M$ be a model that satisfies this formula. Let $v$ be an interpretation for $\vec{x}$ such that $M$ under $v$ satisfies $\forall \vec{y} \varphi(\vec{x}, \vec{y})$. Then it is not hard to argue that the submodel obtained by picking only the elements used in the interpretation of $\vec{x}$ (i.e. $v(\vec{x})$ ), and projecting each relation to this smaller set, satisfies the formula $\exists \vec{x} \forall \vec{y} \varphi(\vec{x}, \vec{y})$ as well [6]. Hence a model of size at most $k$ always exists that satisfies $\varphi$, if the formula is satisfiable, where $k$ is the size of the vector of existentially quantified variables $\vec{x}$. This bounded model property extends to when constants are present as well (the submodel should include all the constants) but fails when more than two functions are present. Satisfiability hence reduces to propositional satisfiability, and this class is also called the effectively propositional class, and SMT solving for this class exists [8].

The decidable fragment of STRAND is fashioned after a similar but more complex argument. Given a subset of nodes of a model, the subset itself may not form a valid graph/data-structure. We define a notion of submodels that allows us to extract proper subgraphs that contain certain nodes of the model. However, the relations (edges) in the submodel will not be the projection of edges in the larger model. Consequently, the submodel may not satisfy a formula, even though the larger model does.

We define a notion called satisfiability-preserving embeddings that allows us to identify when a submodel $S$ of $T$ is such that, whenever $T$ satisfies $\psi$ under some interpretation of the data-logic, $S$ can inherit values from $T$ to satisfy $\psi$ as well. This is consider- 
ably more complex and is the main technical contribution of the paper. We then build decision procedures to check the minimal models according to this embedding relation.

\section{Recursive data-structures}

We now define recursive data-structures using a formalism that defines the nodes and edges using MSO formulas over a regular set of trees. Intuitively, a set of data-structures is defined by taking a regular class of trees that acts as a skeleton over which the datastructure will be defined. The precise set of nodes of the tree that corresponds to the nodes of the data-structure, and the edges between these nodes (which model pointer fields) will be captured using MSO formulas over these trees. We call such classes of datastructures recursively defined data-structures.

Recursively defined data-structures are very powerful mechanisms for defining invariants of data-structures. The notion of graph types [14] is a very similar notion, where again data-structure invariants are defined using a tree-backbone but where edges are defined using regular path expressions. Graph types can be modeled directly in our framework; in fact, our formalism is more powerful.

The framework of recursively defined data-structures is also interesting because they define classes of graphs that have a decidable monadic second-order theory. In other words, given a class $\mathcal{C}$ of recursively defined data-structures, the satisfiability problem for MSO formulas over $\mathcal{C}$ (i.e. the problem of checking, given $\varphi$, whether there is some structure $R \in \mathcal{C}$ that satisfies $\varphi$ ) is decidable. The decision procedure works by interpreting the MSO formula on the tree-backbone of the structures. In fact, our framework can capture all graphs definable using edge-replacement grammars, which are one of the most powerful classes of graphs known that have a decidable MSO theory [10].

\subsection{Graphs and monadic second-order logics}

A labeled (directed) graph $G$ over a finite set of vertex-labels $L_{v}$ and a finite set of edge labels $L_{e}$ is a 6-tuple, $G=\left(V, E, \mu, \nu, L_{v}\right.$, $\left.L_{e}\right)$, where $V$ is a non-empty finite set of nodes, $E \subseteq V \times V$ is a set of edges, $\mu: V \rightarrow 2^{L_{v}}$ assigns a subset of labels to each vertex, and $\nu: E \rightarrow 2^{L_{e}}$ assigns a subset of labels to each edge.

Monadic second-order logic (MSO) on graphs over the labels $\left(L_{v}, L_{e}\right)$ is the standard MSO on structures of the form $\left(U, E,\left\{Q_{a}\right\}_{a \in L_{v}},\left\{E_{b}\right\}_{b \in L_{e}}\right)$ where $U$ represents the universe, $E$ is a binary relation capturing the edge relation, $Q_{a}$ is a monadic predicate that captures all nodes whose labels contain $a$, and $E_{b}$ is a binary relation that captures all edges whose label contain $b$ (note that $E_{b} \subseteq E$, for every $b \in L_{e}$ ). However, we also allow Boolean variables and quantification over them ${ }^{1}$.

Let us fix a countable set of first-order variables $F V$ (first-order variables will be denoted by $s, t$, etc.) and a countable set of setvariables $S V$ (set-variables will be denoted by $S, T$, etc.). Let us also fix a countable set of Boolean variables $B V$ (denoted by $p, q$, etc.) The syntax of the logic is:

$$
\begin{array}{r}
\varphi:=p\left|Q_{a}(s)\right| E(s, t)\left|E_{b}(s, t)\right| s=t|s \in S| \\
\varphi \vee \varphi|\neg \varphi| \exists s . \varphi|\exists p . \varphi| \exists S . \varphi
\end{array}
$$

where $a \in L_{v}, b \in L_{e}, s, t \in F V, S \in S V$, and $p \in B V$.

\subsection{Recursively defined data-structures}

Let $\Sigma$ be a finite alphabet. For any $k \in \mathbb{N}$, let $[k]$ denote the set $\{1, \ldots k\}$.

\footnotetext{
${ }^{1}$ Classical definitions of MSO do not allow such Boolean quantification, but we will find it useful in our setting. These variables can be easily removed; e.g. instead of quantifying over a Boolean variable $p$, we can quantify over a set $X$ and convert every occurrence of $p$ to a formula that expresses that $X$ is empty.
}

A $k$-ary $\Sigma$-labeled tree is a pair $(V, \lambda)$, where $V \subseteq[k]^{*}$, and $V$ is non-empty and prefix-closed, and $\lambda: V \rightarrow \Sigma$. The edges of the tree are implicitly defined: that is $u . i$ is the $i$ 'th child of $u$, for every $u, u . i \in V$, where $u \in[k]^{*}$ and $i \in[k]$. Trees are seen as graphs with $\Sigma$-labeled vertices and edge relations $E_{i}(x, y)$ that define the $i$ 'th-child edges. Monadic second-order logic over trees is the MSO logic over these graphs.

Formally, we define classes of recursively defined data-structures as follows.

Definition 3.1. A class $\mathcal{C}$ of recursively defined data-structures is specified by a tuple $\mathcal{R}=\left(\psi_{T r}, \psi_{U},\left\{\alpha_{a}\right\}_{a \in L_{v}},\left\{\beta_{b}\right\}_{b \in L_{e}}\right)$, where $\psi_{T r}$ is an MSO sentence, $\psi_{U}$ is a unary predicate defined in MSO, and each $\alpha_{a}$ and $\beta_{b}$ are monadic and binary predicates defined using MSO, where all MSO formulas are over $k$-ary trees, for some $k \in \mathbb{N}$, .

Let $\mathcal{R}=\left(\psi_{T r}, \psi_{U},\left\{\alpha_{a}\right\}_{a \in L_{v}},\left\{\beta_{b}\right\}_{b \in L_{e}}\right)$ and $T$ be a $k$-ary $\Sigma$-labeled tree. Then $T=\left(V,\left\{E_{i}\right\}_{i \in[k]}\right)$ defines (according to $\mathcal{R}$ ) a graph $\operatorname{Graph}(T)=\left(N, E, \mu, \nu, L_{v}, L_{e}\right)$ defined as follows:

- $N=\left\{s \in V \mid \psi_{U}(s)\right.$ holds in $\left.T\right\}$

- $E=\left\{\left(s, s^{\prime}\right) \mid \beta_{b}\left(s, s^{\prime}\right)\right.$ holds in T for some $\left.b \in L_{e}\right\}$

- $\mu(s)=\left\{a \in L_{v} \mid \alpha_{a}(s)\right.$ holds in $\left.T\right\}$

- $\nu\left(\left(s, s^{\prime}\right)\right)=\left\{b \in L_{e} \mid \beta_{b}\left(s, s^{\prime}\right)\right.$ holds in $\left.T\right\}$.

The class of graphs defined by $\mathcal{R}$ is the set $\operatorname{Graph}(\mathcal{R})=$ $\left\{\operatorname{Graph}(T) \mid T \models \psi_{T r}\right\}$.

EXAMPLE 3.2. Let us define a class of recursive data-structures that consists of trees where the leaves of the tree are connected by a linked list. The class of trees will be the class of binary trees (with edges $E_{1}$ and $E_{2}$ representing left- and right-child relations), and we define the next-edge relation for the list using an MSO predicate:

$$
E_{\text {next }}(s, t) \equiv \operatorname{leaf}(s) \wedge \operatorname{leaf}(t) \wedge \exists z_{1}, z_{2}, z_{3}\left(E_{1}\left(z_{3}, z_{1}\right) \wedge\right.
$$
$E_{2}\left(z_{3}, z_{2}\right) \wedge$ RightMostPath $\left.\left(z_{1}, s\right) \wedge \operatorname{LeftMostPath}\left(z_{2}, t\right)\right)$

where leaf $(x)$ is a subformula that checks if $x$ is a leaf, and RightMostPath $(x, y)$ (and LeftMostPath $(x, y)$ ) is a formula that checks if $y$ is in the right-most (left-most, respectively) path from $x$.

\section{STRAnD: A logic over heap structures and data}

\subsection{Definition of STRAND}

We now introduce our logic STRAND ("STRucture ANd Data"). STRAND is a two-sorted logic interpreted on program heaps with both locations and their carried data. Given a first-order theory $\mathcal{D}$ of sort Data, and given $\mathcal{L}$, a monadic second-order (MSO) theory over $\left(L_{v}, L_{e}\right)$-labeled graphs, of sort $L o c$, the syntax of STRAND is presented in Figure 1. STRAND is defined over the two-sorted signature $\Gamma(\mathcal{D}, \mathcal{L})=\operatorname{Sig}(\mathcal{D}) \cup \operatorname{Sig}(\mathcal{L}) \cup\{$ data $\}$, where data is a function of sort $L o c \rightarrow D a t a$. STRAND formulas are of the form $\exists \vec{x} \forall \vec{y} \varphi(\vec{x}, \vec{y})$, where $\vec{x}$ and $\vec{y}$ are $\exists D V a r$ and $\forall$ DVar, respectively, of sort Loc (we also refer to both as DVar), $\varphi$ is an MSO formula with atomic formulas of the form either $\gamma\left(e_{1}, \ldots, e_{n}\right)$ or $\alpha\left(v_{1}, \ldots, v_{n}\right) . \gamma\left(e_{1}, \ldots, e_{n}\right)$ is an atomic $\mathcal{D}$-formula in which the data carried by $L o c$-variables can be referred as $\operatorname{data}(x)$ or $\operatorname{data}(y) . \alpha\left(v_{1}, \ldots, v_{n}\right)$ is just an atomic formula from $\mathcal{L}$. Note that additional variables are allowed in $\varphi(\vec{x}, \vec{y})$, both first-order and second-order, but $\gamma\left(e_{1}, \ldots, e_{n}\right)$ is only allowed to refer to $\vec{x}$ and $\vec{y}$.

A model for STRAND is a structure $\mathcal{M}=\left\langle\mathcal{M}_{\text {Loc }}, \mathcal{M}_{\text {Data }}\right.$, $\left.M_{\text {map }}\right\rangle . \mathcal{M}_{L o c}$ is an $\mathcal{L}$-model (i.e. a labeled graph) with $M_{L o c}$ as the underlying set of nodes, and $\mathcal{M}_{\text {Data }}$ is a $\mathcal{D}$-model with $M_{\text {Data }}$ as the underlying set. $M_{m a p}$ is an interpretation for the function data of sort $M_{L o c} \rightarrow M_{D a t a}$. The semantics of STRAND formulas is the natural extension of the logics $\mathcal{L}$ and $\mathcal{D}$. 


\begin{tabular}{|c|c|c|c|}
\hline$\exists$ DVar & $x$ & $\epsilon$ & $L o c$ \\
\hline$\forall$ DVar & $y$ & $\in$ & $L o c$ \\
\hline GVar & $z$ & $\in$ & $L o c$ \\
\hline Variable & $v$ & $::=$ & $x|y| z$ \\
\hline Set - Variable & $S$ & $\in$ & $2^{\operatorname{Loc}}$ \\
\hline Constant & $c$ & $\epsilon$ & $\operatorname{Sig}(\mathcal{D})$ \\
\hline Function & $g$ & $\in$ & $\operatorname{Sig}(\mathcal{D})$ \\
\hline $\mathcal{D}$-Relation & $\gamma$ & $\in$ & $\operatorname{Sig}(\mathcal{D})$ \\
\hline $\mathcal{L}$-Relation & $\alpha$ & $\in$ & $\operatorname{Sig}(\mathcal{L})$ \\
\hline Expression & $e$ & $::=$ & $\operatorname{data}(x)|\operatorname{data}(y)| c \mid g\left(e_{1}, \ldots, e_{n}\right)$ \\
\hline AFormula & $\varphi$ & $::=$ & $\begin{array}{l}\gamma\left(e_{1}, \ldots, e_{n}\right) \mid \alpha\left(v_{1}, \ldots, v_{n}\right) \\
|\neg \varphi| \varphi_{1} \wedge \varphi_{2} \mid \varphi_{1} \vee \varphi_{2} \\
|\exists z . \varphi| \forall z . \varphi|\exists S . \varphi| \forall S . \varphi\end{array}$ \\
\hline$\forall$ Formula & $\omega$ & $::=$ & $\varphi \mid \forall y \cdot \omega$ \\
\hline Formula & $\psi$ & $::=$ & $\omega \mid \exists x . \psi$ \\
\hline
\end{tabular}

Figure 1. Syntax of STRAND

We will refer to an $\mathcal{L}$-model as a graph-model. A data-extension of a graph model $\mathcal{M}_{\text {Loc }}$ is a STRAND model $\left\langle\mathcal{M}_{\text {Loc }}, \mathcal{M}_{\text {Data }}, M_{\text {map }}\right\rangle$.

Undecidability. STRAND is an expressive logic, as we will show below, but it is undecidable in general, even if both its underlying theories $\mathcal{D}$ and $\mathcal{L}$ are decidable. Let $\mathcal{D}$ be linear integer arithmetic and $\mathcal{L}$ be the standard MSO logic over lists. It is easy to model an execution of a 2-counter machine using a list with integers. Each configuration is represented by two adjacent nodes, which are labeled by the current instruction. The data fields of the two nodes hold the value of the two registers, respectively. Then a halting computation can be expressed by a STRAND formula. Hence the satisfiability of the STRAND logic is undecidable, even though the underlying logics $\mathcal{L}$ and $\mathcal{D}$ are decidable.

\subsection{Examples}

We now show various examples to illustrate the expressiveness of STRAND. We sometimes use $d()$ instead of data(), for brevity.

EXAMPLE 4.1 (Binary search tree). In STRAND, $a$ binary search tree (BST) is interpreted as a binary tree data structure with an additional key field for each node. The keys in a BST are always stored in such a way as to satisfy the binary-search-tree property, expressed in STRAND as follows:

$$
\begin{aligned}
& \text { leftsubtree }\left(y_{1}, y_{2}\right) \equiv \exists z\left(\text { left }\left(y_{1}, z\right) \wedge z \rightarrow^{*} y_{2}\right) \\
& \quad \text { rightsubtree }\left(y_{1}, y_{2}\right) \equiv \exists z\left(\operatorname{right}\left(y_{1}, z\right) \wedge z \rightarrow^{*} y_{2}\right) \\
& \psi_{\text {bst }} \equiv \forall y_{1} \forall y_{2}\left(\left(\text { leftsubtree }\left(y_{1}, y_{2}\right) \Rightarrow d\left(y_{2}\right)<d\left(y_{1}\right)\right) \wedge\right. \\
& \left(\left(\text { rightsubtree }\left(y_{1}, y_{2}\right) \Rightarrow d\left(y_{1}\right) \geq d\left(y_{2}\right)\right)\right)
\end{aligned}
$$

Note that $\psi_{b s t}$ has an existentially quantified variable $z$ in GVar after the universal quantification of $y_{1}, y_{2}$. However, as $z$ is a structural quantification (whose data-field cannot be referred to), this formula is in STRAND.

EXAMPLE 4.2 (Two disjoint lists). In separation logic[26], a novel binary operator $*$, or separating conjunction, is defined to assert that the heap can be split into two disjoint parts where its two arguments hold, respectively. Such an operator is useful in reasoning with frame conditions in program verification. Thanks to the powerful expressiveness of MSO logic, the separating conjunction is also expressible in STRAND. For example, $\left(\right.$ head $_{1} \rightarrow^{*}$ tail $\left._{1}\right) *\left(\right.$ head $_{2} \rightarrow^{*}$ tail $\left._{2}\right)$ states, in separation logic, that there are two disjoint lists such that one list is from head $_{1}$ to tail ${ }_{1}$, and the other is from head to tail $_{2}$. This formula can be written in STRAND as:

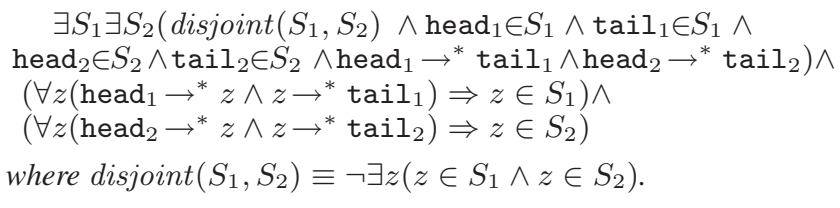

\section{Deciding STRAND fragments}

\subsection{Removing existential quantification:}

Given a STRAND formula $\exists \vec{x} \forall \vec{y} \varphi(\vec{x}, \vec{y})$ over a class of recursively defined data-structures $\mathcal{R}=\left(\psi_{T r}, \psi_{U},\left\{\alpha_{a}\right\}_{a \in L_{v}},\left\{\beta_{b}\right\}_{b \in L_{e}}\right)$, we can transform this to an equisatisfiable formula $\forall \vec{x} \forall \vec{y} \varphi^{\prime}(\vec{x}, \vec{y})$ over a different class of recursive data-structures $\mathcal{R}^{\prime}$, where datastructures in $\mathcal{R}^{\prime}$ are data-structures in $\mathcal{R}$ with new unary predicates that give a valuation for the variables in $\vec{x}$. We won't define this formally, but this is an easy transformation: we modify $\psi_{T r}$ to accept trees with extra labelings $a_{i}$ that give (an arbitrary) singleton valuation of each $x_{i} \in \vec{x}$ that satisfies $\psi_{U}$, and introduce new unary predicates $\operatorname{Val}_{i}(x)=Q_{a_{i}}(x)$, and define $\varphi^{\prime}(\vec{x}, \vec{y})$ to be $\left(\wedge_{i} \operatorname{Val}_{i}\left(x_{i}\right)\right) \Rightarrow \varphi(\vec{x}, \vec{y})$. It is easy to see there is a graph in $\operatorname{Graph}(\mathcal{R})$ that satisfies $\exists \vec{x} \forall \vec{y} \varphi(\vec{x}, \vec{y})$ iff there is a graph in $\operatorname{Graph}\left(\mathcal{R}^{\prime}\right)$ that satisfies $\forall \vec{x} \forall \vec{y} \varphi^{\prime}(\vec{x}, \vec{y})$. The latter is a STRAND formula with no existential quantification of variables whose data is referred to by the formula. Let us refer to these formulas with no leading existential quantification on data-variables as universal STRAND formulas; we will now outline techniques to solve the satisfiability problem of a certain class of universal STRAND formulas.

\subsection{Submodels}

Let us fix a class of recursively defined data-structures $\mathcal{R}=$ $\left(\psi_{T r}, \psi_{U},\left\{\alpha_{a}\right\}_{a \in L_{v}},\left\{\beta_{b}\right\}_{b \in L_{e}}\right)$ for the rest of this section.

We first need to define the notion of submodels of a model. The definition of a submodel will depend on the particular class of recursively defined data-structures we are working with, since we want to exploit the tree-representation of the models, which in turn will play a crucial role in deciding fragments of STRAND, as it will allow us to check satisfiability-preserving embeddings. In fact, we will define the submodel relation between trees that satisfy $\psi_{T r}$.

DEFINITION 5.1. Let $T=(V, \lambda)$ be a tree that satisfies $\psi_{T r}$, and let $S \subseteq V$. Then we say that $S$ is a valid subset of $V$ if the following hold:

- $S$ is non-empty, and least-ancestor closed (i.e. for any $s, s^{\prime} \in$ $S$, the least common ancestor of $s$ and $s^{\prime}$ in $T$ also belongs to $S)$.

- The subtree defined by $S$, denoted Subtree $(T, S)$, is the tree with nodes $S$, and where the i'th child of a node $u \in S$ is the (unique) node $u^{\prime} \in S$ closest to $u$ that is in the subtree rooted at the $i$ 'th child of $u$. (This is uniquely defined since $S$ is least-ancestor closed.) Then we require that Subtree $(T, S)$ also satisfies $\psi_{T r}$.

- We also require that for every $s \in S$, if $\psi_{U}(s)$ holds in Subtree $(T, S)$, then $\psi_{U}(s)$ holds in $T$ as well.

A tree $T^{\prime}=\left(V^{\prime}, \lambda^{\prime}\right)$ is said to be a submodel of $T=(V, \lambda)$ if there is a valid subset $S$ of $V$ such that $T^{\prime}$ is isomorphic to Subtree $(T, S)$.

Note that a submodel is necessarily a valid data-structure.

Intuitively, $T^{\prime}=\left(V^{\prime}, \lambda^{\prime}\right)$ is a submodel of $T=(V, \lambda)$ if the vertices of $T^{\prime}$ can be embedded in $T$, preserving the treestructure. The nodes of the $\operatorname{Graph}\left(T^{\prime}\right)$, are a subset of the nodes of $\operatorname{Graph}(T)$ (because of the last condition in the definition of a submodel), and, given a valid subset $S$, there is in fact an injective mapping from the nodes of $\operatorname{Graph}\left(T^{\prime}\right)$ to $\operatorname{Graph}(T)$. For technical 
convenience, we will work with valid subsets mostly, as fixing the precise embedding helps in the decision procedures.

\subsection{Structural abstractions of STRAND formulas}

Let $\psi=\forall \vec{y} \varphi(\vec{y})$ be a universal STRAND formula.

We now define the structural abstraction of $\psi$ as follows. Let $\gamma_{1}, \gamma_{2}, \ldots, \gamma_{r}$ be the atomic relational formulas of the data-logic in $\varphi$. Note that each of these relational formulas will be over the data fields of variables in $\vec{y}$ only (since the data-logic is restricted to working over the terms $\operatorname{data}(y)$, where $y \in \vec{y}$ ).

Consider evaluating $\psi$ over a particular model. After fixing a particular valuation of $\vec{y}$, notice that the data-relations $\gamma_{i}$ get all fixed, and evaluate to true or false. Moreover, once the values of $\gamma_{i}$ are fixed, the rest of the formula is purely structural in nature. Now, if $\psi$ is to hold in the model, then no matter how we choose to evaluate $\vec{y}$ over the nodes of the model, the $\gamma_{i}$ relations must evaluate to true or false in such a way that $\varphi$ holds.

Since we want, in the first phase, to ignore the data-constraints entirely, we will abstract $\psi$ using a purely structural formula by using Boolean variables $b_{1}, \ldots b_{r}$ instead of the data-relations $\gamma_{1}, \gamma_{2}, \ldots, \gamma_{r}$. However, since these Boolean variables get determined only after the valuation of $\vec{y}$ gets determined, and since we are solving for satisfiability, we existentially quantify over these Boolean variables and quantify them after the quantification of $\vec{y}$. Formally $^{2}$,

DEFINITION 5.2. Let $\psi=\forall \vec{y} \varphi(\vec{y})$ be a universal STRAND formula, and let the atomic relational formulas of the data-logic that occur in $\varphi$ be $\gamma_{1}, \gamma_{2}, \ldots, \gamma_{r}$. Then its structural abstraction $\widehat{\psi}$ is defined as the pure MSO formula on graphs:

$$
\forall \vec{y} \exists b_{1} \ldots b_{r} \varphi^{\prime}(\vec{y}, \vec{b})
$$

where $\varphi^{\prime}$ is $\varphi$ with every occurrence of $\gamma_{i}$ replaced with $b_{i}$.

For example, consider the sortedness formula $\psi_{\text {sorted }}$ from Example 2.1. Then

$$
\begin{gathered}
\widehat{\psi}_{\text {sorted }}: \quad \forall y_{1} \forall y_{2} \exists b_{1}\left(d(\text { head })=c_{1} \wedge d(\text { tail })=c_{2} \wedge\right. \\
\left(\left(y_{1} \rightarrow^{*} y_{2}\right) \Rightarrow b_{1}\right)
\end{gathered}
$$

Note that each Boolean variable $b_{i}$ replaces an atomic relational formula $\gamma_{i}$, where $\gamma_{i}$ places some data-constraint on the data-fields of some of the universally quantified variables.

The following proposition is obvious; it says that if a universal STRAND formula $\psi$ is satisfiable, then so is its structural abstraction $\widehat{\psi}$. The proposition is true because the values for the Boolean variables can be set in the structural abstraction precisely according to how the relational formulas $\gamma_{i}$ evaluate in $\psi$ :

Proposition 5.3. Let $\psi=\forall \vec{y} \varphi(\vec{y})$ be a universal STRAND formula, and $\widehat{\psi}$ be its structural abstraction. If $\psi$ is satisfiable over a set of recursive data-structures $\mathcal{R}$, then the MSO formula on graphs (with no constraints on data) $\widehat{\psi}$ is also satisfiable over $\mathcal{R}$.

\footnotetext{
${ }^{2}$ The definition of structural abstractions can be strengthened in two ways. First, if $\gamma_{i}$ and $\gamma_{j}$ are of the same arity and over $\vec{z}$ and $\overrightarrow{z^{\prime}}$, respectively, and further uniformly replacing $z_{i}$ with $z_{i}^{\prime}$ in $\gamma_{i}$ yields $\gamma^{\prime}$, then we can express the constraint $\left(\left(\overrightarrow{z_{i}}=\overrightarrow{z_{i}}{ }^{\prime}\right) \Rightarrow\left(b_{i} \Leftrightarrow b_{j}\right)\right)$, in the inner formula $\varphi^{\prime}$. Second, if a constraint $\gamma_{i}$ involves only existentially quantified variables in $\vec{x}$, then we can move the quantification of $b_{i}$ outside the universal quantification. Doing these steps gives a more accurate structural abstraction, and in practice, restricts the number of models created. We use these more precise abstractions in the experiments, but use the less precise abstractions in the theoretical narrative. The proofs in this section, however, smoothly extend to the more precise abstractions.
}

\subsection{Satisfiability-preserving embeddings}

We are now ready to define satisfiability-preserving embeddings using structural abstractions. Given a model defined by a tree $T=$ $(V, \lambda)$ satisfying $\psi_{T r}$, and a valid subset $S \subseteq V$, and a universal STRAND formula $\psi$, we would like to define the notion of when the submodel defined by $S$ satisfiability-preservingly embeds in the model. The most crucial requirement for the definition is that if $S$ satisfiability-preservingly embeds in $T$, then we require that if there is a data-extension of $\operatorname{Graph}(T)$ that satisfies $\psi$, then the nodes of the submodel defined by $S, \operatorname{Graph}(\operatorname{Subtree}(T, S))$, can inherit the data-values and also satisfy $\psi$. The notion of structural abstractions defined above allows us to define such a notion.

Intuitively, if a model satisfies $\psi$, then it would satisfy $\widehat{\psi}$ too, as for every valuation of $\vec{y}$, there is some way it would satisfy the atomic data-relations, and using this we can pull out a valuation for the Boolean variables to satisfy $\widehat{\psi}$ (as in the proof of Proposition 5.3 above). Now, since the data-values in the submodel are inherited from the larger model, the atomic data-relations would hold in the same way as they do in the larger model. However, the submodel may not satisfy $\psi$ if the conditions on the truth- and false-hood of these atomic relations demanded by $\psi$ are not the same.

For example, consider a list and a sublist of it. Consider a formula that demands that for any two successor elements $y_{1}, y_{2}$ in the list, the data-value of $y_{2}$ is the data-value of $y_{1}$ incremented by 1 (as in the successor example in Section 2):

$$
\psi \equiv \forall y_{1} \forall y_{2}\left(\left(y_{1} \rightarrow y_{2}\right) \Rightarrow\left(d\left(y_{2}\right)=d\left(y_{1}\right)+1\right)\right)
$$

Now consider two nodes $y_{1}$ and $y_{2}$ that are successors in the sublist but not successors in the list. The list hence could satisfy the formula by setting the data-relation $\gamma: d\left(y_{2}\right)=d\left(y_{1}\right)+1$ to false. Since the sublist inherits the data values, $\gamma$ would be false in the sublist as well, but the sublist will not satisfy the formula $\psi$. We hence want to ensure that no matter how the larger model satisfies the formula using some valuation of the atomic datarelations, the submodel will be able to satisfy the formula using the same valuation of the atomic data-relations. This leads us to the following definition:

DEFINITION 5.4. Let $\psi=\forall \vec{y} \varphi(\vec{y})$ be a universal STRAND formula, and let its structural abstraction be $\widehat{\psi}=\forall \vec{y} \exists \vec{b} \varphi^{\prime}(\vec{y}, \vec{b})$. Let $T=(V, \lambda)$ be a tree that satisfies $\psi_{T r}$, and let a submodel be defined by $S \subseteq V$. Then $S$ is said to satisfiability-preservingly embed into $T$ wrt $\psi$ if for every possible valuation of $\vec{y}$ over the elements of $S$, and for every possible Boolean valuation of $\vec{b}$, if $\varphi^{\prime}(\vec{y}, \vec{b})$ holds in the graph defined by $T$ under this valuation, then the submodel defined by $S$, Graph $(\operatorname{Subtree}(T, S))$, also satisfies $\varphi^{\prime}(\vec{y}, \vec{b})$ under the same valuation.

The satisfiability-preserving embedding relation can be seen as a partial order over trees (a tree $T^{\prime}$ satisfiability-preservingly embeds into $T$ if there is a subset $S$ of $T$ such that $S$ satisfiabilitypreservingly embeds into $T$ and $\operatorname{Subtree}(T, S)$ is isomorphic to $\left.T^{\prime}\right)$; it is easy to see that this relation is reflexive, anti-symmetric and transitive.

It is now not hard to see that if $S$ satisfiability-preservingly embeds into $T$ wrt $\psi$, and $\operatorname{Graph}(T)$ satisfies $\psi$, then Graph (Subtree( $T, S)$ ) also necessarily satisfies $\psi$, which is the main theorem we seek.

THEOREM 5.5. Let $\psi=\forall \vec{y} \varphi(\vec{y})$ be universal STRAND formula. Let $T=(V, \lambda)$ be a tree that satisfies $\psi_{T r}$, and $S$ be a valid subset of $T$ that satisfiability-preservingly embeds into $T$ wrt $\psi$. Then, if there is a data-extension of Graph $(T)$ that satisfies $\psi$, then there is a data-extension of $\operatorname{Graph}(\operatorname{Subtree}(T, S))$ that satisfies $\psi$. 
Notice that the above theorem crucially depends on the formula being universal over data-variables. For example, if the formula was of the form $\forall y_{1} \exists y_{2} \gamma\left(y_{1}, y_{2}\right)$, then we would have no way of knowing which nodes are used for $y_{2}$ in the data-extension of $\operatorname{Graph}(T)$ to satisfy the formula. Without knowing the precise meaning of the data-predicates, we would not be able to declare that whenever a data-extension of $\operatorname{Graph}(T)$ is satisfiable, a dataextension of a strict submodel $S$ is satisfiable (even over lists).

The above notion of satisfiability preserving embeddings is the property that will be used to decide if a formula falls into our decidable fragment.

\subsection{STRAND ${ }_{d e c}^{\text {sem }}$ : A semantic decidable fragment of STRAND}

We are now ready to define $\mathrm{STRAND}_{\text {dec }}^{\text {sem }}$, the most general decidable fragment of STRAND in this paper. This fragment is semantically defined (but syntactically checkable, as we show below), and intuitively contains all STRAND formulas that have a finite number of minimal models with respect to the partial-order defined by satisfiability-preserving embeddings.

Formally, let $\psi=\forall \vec{y} \varphi(\vec{y})$ be a universal STRAND formula, and let $T=(V, \lambda)$ be a tree that satisfies $\psi_{T r}$. Then we say that $T$ is $a$ minimal model with respect to $\psi$ if there is no strict valid subset $S$ of $V$ that satisfiability-preservingly embeds in $T$.

DEFINITION 5.6. Let $\mathcal{R}$ be a recursively defined set of datastructures.

A universal formula $\psi=\forall \vec{y} \varphi(\vec{y})$ is in $\mathrm{STRAND}_{\text {dec }}^{\text {sem }}$ iff the number of minimal models with respect to $\mathcal{R}$ and $\psi$ is finite.

A STRAND formula of the form $\psi=\exists \vec{x} \forall \vec{y} \varphi(\vec{x}, \vec{y})$ is in $\mathrm{STRAND}_{\text {dec }}^{\text {sem }}$ iff the corresponding equi-satisfiable universal formula $\psi^{\prime}$ over set of data-structure $\mathcal{R}^{\prime}$ (as defined in Section 5.1) is in STRAND dec $^{\text {sem }}$.

We now show that we can effectively check if a STRAND formula belongs to the decidable fragment STRAND dec $^{\text {sem }}$. The idea, intuitively, is to express that a model is a minimal model with respect to satisfiability-preserving embeddings, and then check, using automata theory, that the number of minimal models is finite.

Let $\psi=\forall \vec{y} \varphi(\vec{y})$ be universal STRAND formula, and let its structural abstraction be $\widehat{\psi}=\forall \vec{y} \exists \vec{b} \varphi^{\prime}(\vec{y}, \vec{b})$.

We now show that we can define an MSO formula MinModel $\psi$, such that it holds on a tree $T=(V, \lambda)$ iff $T$ defines a minimal model with respect to satisfiability-preserving embeddings.

Before we do that, we need some technical results and notation. Let $\mathcal{R}=\left(\psi_{T r}, \psi_{U},\left\{\alpha_{a}\right\}_{a \in L_{v}},\left\{\beta_{b}\right\}_{b \in L_{e}}\right)$.

We first show that any (pure) MSO formula $\delta$ on $\left(L_{v}, L_{e}\right)$ labeled graphs can be interpreted on trees. Formally, we show that any (pure) MSO formula $\delta$ on $\left(L_{v}, L_{e}\right)$-labeled graphs can be transformed syntactically to a (pure) MSO formula $\delta^{\prime}$ on trees such that for any tree $T=(V, \lambda), \operatorname{Graph}(T))$ satisfies $\delta$ iff $T$ satisfies $\delta^{\prime}$.

This is not hard to do, since the graph is defined using MSO formulas on the trees, and we can adapt these definitions to work over the tree instead. The transformation is given by the following function interpret; the predicates for edges, and the predicates that check vertex labels and edges labels are transformed according to their definition, and all quantified variables are restricted to quantify over nodes that satisfy $\psi_{U}$.

- $\operatorname{interpret}(p)=p$

- interpret $\left(Q_{a}(s)\right)=\alpha_{a}(s)$, for every $a \in L_{v}$

- interpret $(E(s, t))=\bigvee_{b \in L_{e}} \beta_{b}(s, t)$

- interpret $\left(E_{b}(s, t)\right)=\beta_{b}(s, t)$, for every $b \in L_{e}$

- $\operatorname{interpret}(s=t)=(s=t)$

- $\operatorname{interpret}(s \in W)=s \in W$

- $\operatorname{interpret}\left(\varphi_{1} \vee \varphi_{2}\right)=\operatorname{interpret}\left(\varphi_{1}\right) \vee \operatorname{interpret}\left(\varphi_{2}\right)$
- interpret $(\neg \varphi)=\neg($ interpret $(\varphi))$

- interpret $(\exists s . \varphi)=\exists s .\left(\psi_{U}(s) \wedge \operatorname{interpret}(\varphi)\right)$

- interpret $(\exists W . \varphi)=\exists W .\left(\left(\forall s .\left(s \in W \Rightarrow \psi_{U}(s)\right)\right) \wedge \operatorname{interpret}(\varphi)\right)$

It is not hard to show that for any formula $\delta$ on $\left(L_{v}, L_{e}\right)$-labeled graphs $\operatorname{Graph}(T)$ satisfies $\delta$ iff $T$ satisfies interpret $(\delta)$.

Now, we give another transformation, that transforms an MSO formula $\delta$ on trees to a formula $\delta^{\prime}(X)$ on trees, over a free setvariable $X$, such that for any tree $T=(V, \lambda)$ and any valid subset $S \subseteq V$, Subtree $(T, S)$ satisfies $\delta$ iff $T$ satisfies $\delta^{\prime}(X)$ when $X$ is interpreted to be $S$. In other words, we can transform a formula that expresses a property of a subtree to a formula that expresses the same property on the subtree defined by the free variable $X$. The transformation is given by the following function tailor; the crucial transformation are the edge-formulas, which has to be interpreted as the edges of the subtree defined by $X$.

- $\operatorname{tailor}_{X}(p)=p$

- tailor $_{X}\left(Q_{a}(s)\right)=Q_{a}(s)$, for every $a \in L_{v}$

- tailor $_{X}\left(E_{i}(s, t)\right)=\exists s^{\prime}\left[E_{i}\left(s, s^{\prime}\right) \wedge s^{\prime} \leq t \wedge\right.$ for every $i \in[k]$. $\left.\forall t^{\prime} .\left(\left(t^{\prime} \in X \wedge s^{\prime} \leq t^{\prime}\right) \Rightarrow t \leq t^{\prime}\right)\right]$,

- tailor $_{X}(s=t)=(s=t)$

- $\operatorname{tailor}_{X}(s \in W)=s \in W$

- $\operatorname{tailor}_{X}\left(\varphi_{1} \vee \varphi_{2}\right)=\operatorname{tailor}\left(\varphi_{1}\right) \vee \operatorname{tailor}\left(\varphi_{2}\right)$

- tailor $_{X}(\neg \varphi)=\neg($ tailor $(\varphi))$

- $\operatorname{tailor}_{X}(\exists s . \varphi)=\exists s .(s \in X \wedge \operatorname{tailor}(\varphi))$

- $\operatorname{tailor}_{X}(\exists W . \varphi)=\exists W .(W \subseteq X \wedge \operatorname{tailor}(\varphi))$

The above transformation satisfies the following property. For any MSO sentence $\delta$ on $k$-ary trees, for any tree $T=(V, \lambda)$ and for any valid subset $S \subseteq V$, Subtree $(T, S)$ satisfies $\delta$ iff $T$ satisfies tailor $_{X}(\delta)$ when $X$ is interpreted to be $S$.

Note that the above transformations can be combined. For any MSO formula $\delta$ on $\left(L_{v}, L_{e}\right)$ labeled graphs, consider the formula tailor $_{X}($ interpret $(\delta))$. Then for any tree $T=(V, \lambda)$ and for any valid subset $S \subseteq V, \operatorname{Graph}(\operatorname{Subtree}(T, S))$ satisfies $\delta$ iff $T$ satisfies tailor $_{X}($ interpret $(\delta))$, where $X$ is interpreted as $S$.

Expressing minimal models in MSO. First, we can also express, with an MSO formula ValidSubModel $(X)$, with a free set variable $X$, that holds in a tree $T=(V, \lambda)$ iff $X$ is interpreted as a valid submodel of $T$ :

$$
\begin{aligned}
& \text { ValidSubModel }(X) \equiv \\
& \forall s, t, u((s \in X \wedge t \in X \wedge \operatorname{lca}(s, t, u)) \Rightarrow u \in X) \wedge \operatorname{tailor}_{X}\left(\psi_{T r}\right) \\
& \wedge\left(\forall s\left(s \in X \wedge \operatorname{tailor}_{X}\left(\psi_{U}(s)\right)\right) \Rightarrow \psi_{U}(s)\right)
\end{aligned}
$$

where $l c a(s, t, u)$ is an MSO formula that checks whether $u$ is the least-common ancestor of $s$ and $t$ in the tree; this expresses the requirements in Definition 5.1.

We are now ready to define the MSO formula on $k$-ary trees MinModel $_{\psi}$ that captures minimal models. Let the structural abstraction of $\psi$ be $\widehat{\psi}=\forall \vec{y} \exists \vec{b} \varphi^{\prime}(\vec{y}, \vec{b})$, then

$$
\begin{aligned}
& \text { MinModel }_{\psi} \equiv \neg \exists X .(\quad \text { ValidSubModel }(X) \wedge \\
& \quad \exists s .(s \in X) \wedge \exists s .(s \notin X) \wedge \\
& \left(\forall \vec { y } \forall \vec { b } \left(\left(\wedge_{y \in \vec{y}}\left(y \in X \wedge \psi_{U}(y)\right) \wedge \operatorname{interpret}\left(\varphi^{\prime}(\vec{y}, \vec{b})\right)\right)\right.\right. \\
& \left.\left.\quad \Rightarrow \operatorname{tailor}_{X}\left(\text { interpret }\left(\varphi^{\prime}(\vec{y}, \vec{b})\right)\right)\right)\right)
\end{aligned}
$$

The above formula when interpreted on a tree $T$ says that there does not exists a set $X$ that defines a non-empty valid strict subset of the nodes of $T$, which defines a model $\operatorname{Graph}(\operatorname{Subtree}(T, X))$ that further satisfies the following: for every valuation of $\vec{y}$ over the nodes of $\operatorname{Graph}(\operatorname{Subtree}(T, S))$ and for every valuation of the Boolean variables $\vec{b}$ such that the structural abstraction of $\varphi$ holds in 
$\operatorname{Graph}(T)$, the same valuation also makes the structural abstraction of $\varphi$ hold in $\operatorname{Graph}(\operatorname{Subtree}(T, S))$.

Note that the above is a pure MSO formula on trees, and encodes the properties required of a minimal model with respect to satisfiability-preserving embeddings. Using the classical logicautomaton connection [6], we can transform the MSO formula MinModel $_{\psi} \wedge \psi_{T r} \wedge \widehat{\psi}$ to a tree automaton that accepts precisely those trees that define data-structures that satisfy the structural abstraction and are minimal models. Since the finiteness of the language accepted by a tree automaton is decidable, we can check whether there are only a finite number of minimal models wrt satisfiability-preserving embeddings, and hence decide membership in the decidable fragment STRAND ${ }_{d e c}^{s e m}$.

THEOREM 5.7. Given a sentence $\exists \vec{x} \forall \vec{y} \varphi(\vec{x}, \vec{y})$, the problem of checking whether the sentence belongs to the fragment $\mathrm{STRAND}_{\text {dec }}^{\text {sem }}$ is decidable.

In fact, we develop, using the tool MoNA, the decision procedure above (see Section 7).

Deciding formulas in STRAND dec $^{\text {sem }}$. We now give the decision procedure for satisfiability of sentences in STRAND dec over a recursively defined class of data-structures. First, we transform the satisfiability problem to that of satisfiability of universal formulas of the form $\psi=\forall \vec{y} \varphi(\vec{y})$. Then, using the formula MinModel $_{\psi}$ described above, and by transforming it to tree automata, we extract the set of all trees accepted by the tree-automaton in order to get the tree-representation of all the minimal models. Note that this set of minimal models is finite, and the sentence is satisfiable iff it is satisfiable in some data-extension of one of these models.

We can now write a quantifier-free formula over the data-logic that asserts that one of the minimal models has a data-extension that satisfies $\psi$. This formula will be a disjunction of $m$ sub-formulas $\eta_{1}, \ldots, \eta_{m}$, where $m$ is the number of minimal models. Each formula $\eta_{i}$ will express that there is a data-extension of the $i$ 'th minimal model that satisfies $\psi$. First, since a minimal model has only a finite number of nodes, we create one data-variable for each of these nodes, and associate them with the nodes of the model. It is now not hard to transform the formula $\psi$ to this model using no quantification. The universal quantification over $\vec{y}$ translates to a conjunction of formulas over all possible valuations of $\vec{y}$ over the nodes of the fixed model. Existential (universal) quantified variables are then "expanded" using disjunction (conjunction, respectively) of formulas for all possible valuations over the fixed model. The edge-relations between nodes in the model are interpreted on the tree using MSO formulas in $\mathcal{R}$, which are then expanded to conditions over the fixed set of nodes in the model. Finally, the data-constraints in the STRAND formula are directly written as constraints in the data-logic.

The resulting formula is a pure data-logic formula without quantification that is satisfiable if and only if $\psi$ is satisfiable over $\mathcal{R}$. This is then decided using the decision procedure for the data-logic.

THEOREM 5.8. Given a sentence $\exists \vec{x} \forall \vec{y} \varphi(\vec{x}, \vec{y})$ over $\mathcal{R}$ in $\mathrm{STRAND}_{d e c}^{\text {sem }}$, the problem of checking whether $\psi$ is satisfiable reduces to the satisfiability of a quantifier-free formula in the datalogic. Since the quantifier-free data-logic is decidable, the satisfiability of $\mathrm{STRAND}_{\text {dec }}^{\text {sem }}$ formulas is decidable.

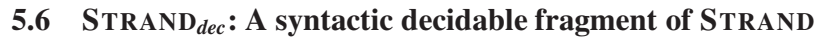

We utilize the semantically defined decidable class in the previous section to define a logic that has a simple syntactic restriction and is entirely decidable. The decidable fragment allows only formulas of the kind $\exists \vec{x} \forall \vec{y} \varphi$ where $\varphi$ has no further quantification. Moreover, some of the structural edge relations $R$ on the data-structure are classified as elastic relations. In $\varphi$, elastic relations are allowed to relate any pair of variables, while non-elastic relations are allowed only to relate existentially quantified variables in $\vec{x}$.

A relation $R$ is elastic if, intuitively, for any model $M$ and a submodel $M^{\prime}$ of $M, R$ holds on a pair of nodes of $M^{\prime}$ iff $R$ holds for the corresponding pair of nodes in $M$.

More formally, let us fix a class of recursively defined datastructures $\mathcal{R}=\left(\psi_{T r}, \psi_{U},\left\{\alpha_{a}\right\}_{a \in L_{v}},\left\{\beta_{b}\right\}_{b \in L_{e}}\right)$. Let $E_{b}$ denote the edge-relation defined by $\beta_{b}$. Then we say $E_{b}$ is elastic if the following holds: for every tree $T=\left(V,\left\{E_{i}\right\}_{i \in[k]}\right)$ satisfying $\psi_{T r}$, for every valid subset $S$ of $V$, and for every pair of nodes $u, v$ in the model $M^{\prime}=\operatorname{Graph}(\operatorname{Subtree}(T, S)), E_{b}(u, v)$ holds in $M^{\prime}$ iff $E_{b}(u, v)$ holds in $\operatorname{Graph}(T)$.

For example, over trees, the $\leq$ relation relating a node with any of its descendants is an elastic relation; however, the relation that relates a node to its parent is not elastic, as we can take two nodes $u$ and $v$ in a subtree $\operatorname{Subtree}(S, T)$ where $u$ is the parent of $v$, but $u$ is not the parent of $v$ in $T$.

We can express the property that $R_{b}$ is elastic in MSO over a particular tree $T$ using the following formula:

$\forall S \forall u \forall v\left(\left(\right.\right.$ ValidSubModel $(S) \wedge u \in S \wedge v \in S \wedge \operatorname{tailor}_{S}\left(\psi_{U}(u)\right)$ $\left.\left.\wedge \operatorname{tailor}_{S}\left(\psi_{U}(v)\right)\right) \Rightarrow\left(\beta_{b}(u, v) \Leftrightarrow \operatorname{tailor}_{S}\left(\beta_{b}(u, v)\right)\right)\right)$

Hence, we can decide whether a relation is elastic or not, by checking the validity of the above formula over all trees satisfying $\psi_{T r}$.

The syntactic decidable fragment $\mathrm{STRAND}_{d e c}$ is defined as the class of all STRAND formulas of the form $\exists \vec{x} \forall \vec{y} \varphi$ such that (a) $\varphi$ has no quantification, (b) every occurrence of an atomic relation in $\varphi$ is of the form $R\left(z_{1}, z_{2}\right)$ where either $R$ is an elastic relation or $z_{1}$ and $z_{2}$ are in $\vec{x}$, or are constants. We can now show:

THEOREM 5.9. Over any class of recursively defined structures $\mathcal{R}$, $\mathrm{STRAND}_{\text {dec }}$ is decidable.

We omit the proof for lack of space; it's gist is as follows. When all relations are elastic, for any valid subset $S$, $\operatorname{tailor}_{S}(\varphi)$ holds on any valuation of variables over $S$ iff $\varphi$ holds on the same valuation over $T$ (since the atomic relations are elastic). Hence the submodel can always inherit the data-values from the model to satisfy the formula. The minimal models with respect to satisfiability-preserving embeddings are hence a subset of the minimal models with respect to the submodel-relation, which we can show is finite. When all relations are not elastic, the proof is much more complex, and relies on the fact that the non-elastic relations define only a finite number of equivalence classes of relationships over $\vec{x}$.

All of verification conditions in our experiments turn out to be in the syntactic decidable class STRAND dec $_{\text {. }}$.

\section{Program Verification Using STRAND}

In this section we show how STRAND can be used to reason about the correctness of programs, in terms of verifying Hoare-triples where the pre- and post-conditions express both the structure of the heap as well as the data contained in them. The pre- and post-conditions that we allow are STRAND formulas that consist of Boolean combinations of the formulas with pure existential or pure universal quantification over the data-variables (i.e. Boolean combinations of formulas of the form $\exists \vec{x} \varphi$ and $\forall \vec{y} \varphi$ ); let us call this fragment STRAND $\exists, \forall$.

Given a straight-line program $P$ that does destructive pointerupdates and data updates, we model a Hoare-triple as a tuple $(\mathcal{R}$, Pre, $P$, Post $)$, where the pre-condition is given by the datastructure constraint $\mathcal{R}$ with the $\mathrm{STRAND}_{\exists, \forall}$ formula Pre, and the post-condition is given by the $\mathrm{STRAND}_{\exists, \forall}$ formula Post (note that structural constraints on the data-structure for the post-condition are also expressed in Post, using MSO logic). 
In this section, we show that given such a Hoare-triple, we can reduce checking whether the Hoare-triple is not valid can be reduced to a satisfiability problem of a STRAND formula over a class of recursively defined data-structures $\mathcal{R}_{P}$. This then allows us to use $\mathrm{STRAND}_{\exists, \forall}$ to verify programs (where, of course, loopinvariants are given by the programmer, which breaks down verification of a program to verification of straight-line code). Intuitively, this reduction augments the structures in $\mathcal{R}$ with extra nodes that could be created during the execution of $P$, and models the trail the program takes by logically defining the configuration of the program at each time instant. Over this trail, we then express that the pre-condition holds and the post-condition fails to hold. We also construct formulas that check if there is any memory access violation during the run of $P$ (e.g. free-ing locations twice, dereferencing a null pointer, etc.).

Syntax of programs. Let us define the syntax of a basic programming language manipulating heaps and data; more complex constructs can be defined by combining these statements appropriately. Let Var be a countable set of pointer variables, $F$ be a countable set of structural pointer fields, and data be a data field. A condition is defined as follows: (for technical reasons, negations are pushed all the way in):

$\psi \in$ Cond $::=\gamma\left(q^{1}\right.$.data $, \ldots, q^{k}$.data $) \mid \neg \gamma\left(q^{1}\right.$.data $, \ldots, q^{k}$.data $)$

$$
|p==q| p \neq q \mid p==\text { nil } \mid p \neq \text { nil }\left|\psi_{1} \wedge \psi_{2}\right| \psi_{1} \vee \psi_{2}
$$

where $p, q, q^{1}, \ldots, q^{k} \in \operatorname{Var}$, and $\gamma$ is a predicate over data values. The set of statements Stmt defined over Var, F, and data is defined as follows:

$$
\begin{gathered}
s \in \text { Stmt }::=p:=\text { new } \mid \text { free }(p)|\operatorname{assume}(\psi)| p:=\text { nil } \mid \\
p:=q|p . f:=q| p:=q . f \mid p \cdot \text { data }:=h\left(q^{1} \text {.data, }, \ldots, q^{k} \text {.data }\right)
\end{gathered}
$$

where $p, q, q^{1}, \ldots, q^{k} \in \operatorname{Var}, f \in F, h$ is a function over data, and $\psi$ is a condition. A program $P$ over Var, $F$, and data is a non empty finite sequence of statements $s_{1} ; s_{2} ; \ldots ; s_{m}$, with $s_{i} \in$ Stmt.

The semantics of a program is the natural one and we skip its definition.

Let $\mathcal{R}$ be a recursive data-structure, Pre, Post be two $\mathrm{STRAND}_{\exists, \forall}, \forall$ formulas, and $P::=s_{1} ; s_{2} ; \ldots ; s_{m}$ be a program. The configuration of the program at any point is given by a heap modeled as a graph, where nodes of the graph are assigned data values. For a program with $m$ statements, let us fix the configurations to be $G_{0}, \ldots, G_{m}$.

The trail. The idea is to capture the entire computation starting from a particular data-structure using a single data-structure. The main intuition is that if we run $P$ over a graph $G_{0} \in \operatorname{Graph}(\mathcal{R})$ then a new class of recursive data-structures $\mathcal{R}_{P}$ will define a graph $G_{\text {trail }}$ which encodes in it $G_{0}$, as well as all the graphs $G_{i}$, for every $i \in[m] . G_{\text {trail }}$ has the nodes of $G_{0}$ plus $m$ other fresh nodes (these nodes will be used to model newly created nodes $P$ creates as well as to hold new data-values of variables that are assigned to in $P$ ). Each of these new nodes are pointed by a distinguished pointer variable new $_{i}$. Initially, these additional nodes are all inactive in $G_{0}$. We build an MSO-defined unary predicate active $_{i}$ that captures at each step $i$ the precise set of active nodes in the heap. To capture the pointer variables at each step of the execution, we define a new unary predicate $p_{i}$, for each $p \in \operatorname{Var}$ and $i \in[0, m]$. Similarly, we create MSO-defined binary predicates $f_{i}$ for each $f \in F$ and $i \in[0, m]$, to capture structural pointer fields at step $i$. The heap $G_{i}$ at step $i$ is hence the graph consisting of all the nodes $x$ of $G_{\text {trail }}$ such that $\operatorname{active}_{i}(x)$ holds true, and the pointers and edges of $G_{i}$ are defined by $p_{i}$ and $f_{i}$ predicates, respectively.
Formally, fix a recursively defined data-structure $\mathcal{R}=$ $\left(\psi_{T r}, \psi_{U},\left\{\alpha_{p}\right\}_{p \in V a r},\left\{\beta_{f}\right\}_{f \in F}\right)$, with a monadic predicate $\alpha_{x n i l}$, which evaluates to a unique NIL node in the data-structure. Then its trail with respect to the program $P$ is defined as $\mathcal{R}_{P}=$ $\left(\psi_{T r}^{\prime}, \psi_{U}^{\prime},\left\{\alpha_{p}^{\prime}\right\}_{p \in V a r^{\prime}},\left\{\beta_{f}^{\prime}\right\}_{f \in F^{\prime}}\right)$ where:

- $\psi_{T r}^{\prime}$ is designed to hold on all trees in which the first subtree of the root satisfies $\psi_{T r}$ and the second child of the root has a chain of $m-1$ nodes where each of them is the second child of the parent.

- $\psi_{U}^{\prime}$ holds true on the root, on all the second child descendent of the root, and on all first child descendent on which $\psi_{U}$ holds true.

- $\operatorname{Var}^{\prime}=\left\{\right.$ new $\left._{i} \mid i \in[m]\right\} \cup\left\{p_{i} \mid p \in \operatorname{Var}, i \in[0, m]\right\}$, and -(1) $\alpha_{\text {new }_{1}}^{\prime}$ holds only on the root, and $\alpha_{\text {new }_{i}}^{\prime}$ holds true only on the $i+1$ 'th descendent of the second child of the root, for every $i \in[m-1]$.

-(2) for every $p \in \operatorname{Var}$ and $i \in[m], \alpha_{p_{0}}^{\prime}=\alpha_{p}$ and $\alpha_{p_{i}}^{\prime}$ is defined as in Figure 2.

- $F^{\prime}=\left\{f_{i} \mid f \in F, i \in[0, m]\right\}$, and for every $f \in F$ and $i \in[m]$, $\beta_{f_{0}}^{\prime}=\beta_{f}$ and $\beta_{f_{i}}^{\prime}$ is defined as in Figure 2.

In Figure 2, the MSO formulas $\alpha_{p_{i}}^{\prime}$ and $\beta_{f_{i}}^{\prime}$ are derived in the natural way from the semantics of the statements, except for the statement $p$.data $:=h\left(q^{1}\right.$.data $, \ldots, q^{k}$.data $)$. Although the semantics for this statement does not involve any structural modification of the graph (it changes only the data value associated $p$ ), we represent this operation by making a new version of the node pointed by $p$ in order to represent explicitly the change for the data value corresponding to that node. We deactivate the node pointed by $p_{i-1}$ and activate the dormant node pointed by $n e w_{i}$. All the edges in the graph and the pointers are rearranged to reflect this exchange of nodes.

In Figure 2, we also define two more MSO formulas, active and error $_{i}$, which are not part of the trail, where the first models the active nodes at step $i$, and the second expresses when an error occurs due to the dereferencing of a variable pointing to xnil, respectively.

Handling data constraints. The trail $\mathcal{R}_{P}$ captures all the structural modifications made to the graph during the execution $P$. However, data constrains entailed by assume statements and dataassignments cannot be expressed in the trail as they are not expressible in MSO. We impose them in the STRAND formula. We define a formula $\varphi_{i}$ for each statement index $i \in[m]$, where if $s_{i}$ is not an assume or a data-assignment statement, then $\varphi_{i}=$ true. Otherwise, there are two cases:

Handling assume assignments. If $s_{i}$ is the statement assume $(\psi)$, then $\varphi_{i}$ is the STRAND formula obtained by adapting the constraint $\varphi$ to the $i$ 'th stage of the trail. This is not hard, but is tedious, and we skip its definition. Constraints on data-variables asserted in the formula using data-logic constraints.

Handling data-assignments. The STRAND formula $\varphi_{i}$ for a data$\overline{\text { assignment statement } p \text {.data }}:=h\left(q^{1}\right.$.data $, \ldots, q^{k}$.data $)$ is:

$$
\begin{aligned}
\varphi_{i} & :=\exists e x, e x_{1}, \ldots, e x_{k} \cdot p_{i}(e x) \wedge \\
& \left(\bigwedge_{i \in[k]} q_{i-1}^{j}\left(e x_{j}\right)\right) \wedge \operatorname{data}(e x)=h\left(\operatorname{data}\left(e x_{1}\right), \ldots, \operatorname{data}\left(e x_{k}\right)\right)
\end{aligned}
$$

which translates $s_{i}$ into STRAND making sure that it refers to the heap at step $i-1$.

Adapting pre- and post-conditions to the trail. The last ingredient that we need is to express the $\mathrm{STRAND}_{\exists, \forall}$ formulas Pre and the negation of the Post on the trail $\mathcal{R}_{P}$. More specifically, we need to 


\begin{tabular}{|c|}
\hline $\begin{array}{l}{[\boldsymbol{p}:=\text { new }]:} \\
\quad \alpha_{p_{i}}^{\prime}(x)=\alpha_{\text {new }_{i}}^{\prime}(x), \quad \alpha_{q_{i}}^{\prime}(x)=\alpha_{q_{i-1}}^{\prime}(x), \quad \forall q \in \text { Var } \backslash\{p\} \\
\quad \beta_{f_{i}}^{\prime}(x, y)=\beta_{f_{i-1}}^{\prime}(x, y), \text { active }_{i}(x)=\text { active }_{i-1}(x) \vee \alpha_{\text {new }_{i}}^{\prime}(x) \\
\quad \text { error }_{i}=\text { false }\end{array}$ \\
\hline$[$ free $(p)]:$ \\
\hline $\begin{aligned} \alpha_{z_{i}}^{\prime}(x)= & \left(\alpha_{z_{i-1}}^{\prime}(x) \wedge\left(\alpha_{x n i l_{i-1}}^{\prime}(x) \vee \neg \alpha_{p_{i-1}}^{\prime}(x)\right)\right) \\
& \vee\left(\alpha_{x n i l_{i-1}}^{\prime}(x) \wedge \neg \alpha_{z_{i-1}}^{\prime}(x)\right)\end{aligned}$ \\
\hline$\beta_{f_{i}}^{\prime}(x, y)=\left(\beta_{f_{i-1}}^{\prime}(x, y) \wedge \neg \alpha_{p_{i-1}}^{\prime}(x)\right)$ \\
\hline$\vee\left(\alpha_{x n i i_{i-1}}^{\prime}(y) \wedge \exists e x . \quad\left(\beta_{f_{i-1}}^{\prime}(x, e x) \wedge \alpha_{p_{i-1}}^{\prime}(e x)\right)\right)$ \\
\hline $\operatorname{active}_{i}(x)=$ active $_{i-1}(x) \wedge \neg \alpha_{p_{i-1}}^{\prime}(x)$ \\
\hline error $_{i}=\exists x .\left(\alpha_{p_{i-1}}^{\prime}(x) \wedge \alpha_{x n i l}^{\prime} l_{i-1}^{\prime}(x)\right)$ \\
\hline$[p:=$ nil $]:$ \\
\hline$\alpha_{p_{i}(x)}^{\prime}=\alpha_{x n i l_{i-1}}^{\prime}(x), \quad \alpha_{z_{i}}^{\prime}(x)=\alpha_{z_{i-1}}^{\prime}(x), \quad \forall z \in \operatorname{Var} \backslash\{p\}$ \\
\hline$\beta_{f_{i}}^{\prime}(x, y)=\beta_{f_{i-1}}^{\prime}(x, y)$, active $_{i}(x)=$ active $_{i-1}(x)$, error $_{i}=\mathrm{false}$ \\
\hline$[p:=q]:$ \\
\hline$\alpha_{p_{i}}^{\prime}(x)=\alpha_{q_{i-1}}^{\prime}(x), \quad \alpha_{z_{i}}^{\prime}(x)=\alpha_{z_{i-1}}^{\prime}(x), \quad \forall z \in(\operatorname{Var} \backslash\{p\})$ \\
\hline$\beta_{f_{i}}^{\prime}(x, y)=\beta_{f_{i-1}}^{\prime}(x, y)$, active $_{i}(x)=$ active $_{i-1}(x)$, error $_{i}=$ false $_{\text {als }}$ \\
\hline$[p \cdot f:=q]:$ \\
\hline$\alpha_{z_{i}}^{\prime}(x)=\alpha_{z_{i-1}}^{\prime}(x), \quad \forall z \in \operatorname{Var}$ \\
\hline$\beta_{f_{i}}^{\prime}(x, y)=\left(\neg \alpha_{p_{i-1}}^{\prime}(x) \wedge \beta_{f_{i-1}}^{\prime}(x, y)\right) \vee\left(\alpha_{p_{i}-1}^{\prime}(x) \wedge \alpha_{q_{i-1}}^{\prime}(y)\right)$ \\
\hline$\alpha_{g_{i}}^{\prime}(x, y)=\alpha_{g_{i-1}}^{\prime}(x, y), \quad \forall g \in(F \backslash\{f\})$ \\
\hline $\operatorname{active}_{i}(x)=\operatorname{active}_{i-1}(x), \quad$ error $_{i}=\exists x .\left(\alpha_{p_{i-1}}^{\prime}(x) \wedge \alpha_{x n i l}^{\prime} l_{i-1}(x)\right)$ \\
\hline$[p:=q \cdot f]:$ \\
\hline$\alpha_{p_{i}(x)}^{\prime}=\exists e x . \quad\left(\alpha_{q_{i-1}}^{\prime}(e x) \wedge \beta_{f_{i-1}}^{\prime}(e x, x)\right)$ \\
\hline \multirow{2}{*}{$\begin{aligned} \alpha_{q_{i}}^{\prime}(x) & =\alpha_{q_{i-1}}^{\prime}(x), \quad \forall q \in(\operatorname{Var} \backslash\{p\}) \\
\beta_{f_{i}}^{\prime}(x, y) & =\beta_{f_{i-1}}^{\prime}(x, y)\end{aligned}$} \\
\hline \\
\hline \multirow{2}{*}{$\begin{aligned} \alpha_{g_{i}}^{\prime}(x, y) & =\alpha_{g_{i-1}}^{\prime}(x, y), \quad \forall g \in(F \backslash\{f\}) \\
\text { active }_{i}(x) & =\operatorname{active}_{i-1}(x), \quad \text { error }_{i}=\exists x . \quad\left(\alpha_{q_{i-1}}^{\prime}(x) \wedge \alpha_{x_{n i l}}^{\prime}(x)\right)\end{aligned}$} \\
\hline \\
\hline$\overline{\operatorname{assume}(\psi)]:}$ \\
\hline \multirow{3}{*}{$\begin{array}{r}\alpha_{q_{i}}^{\prime}(x)=\alpha_{q_{i-1}}^{\prime}(x), \forall q \in \operatorname{Var}, \quad \beta_{f_{i}}^{\prime}(x, y)=\beta_{f_{i-1}}^{\prime}(x, y), \forall f \in F \\
\left.\operatorname{active}_{i}(x)=\text { active }_{i-1}(x), \quad \text { error }_{i}=\underset{p \in \operatorname{Var} \psi}{\exists} \bigvee_{p_{i-1}}(x) \wedge \alpha_{x n i l_{i-1}}^{\prime}(x)\right) \\
\text { where } \operatorname{Var}^{\psi} \text { is the set of all variables occurring in } \psi .\end{array}$} \\
\hline \\
\hline \\
\hline$\overline{\left[p \text {.data }:=\boldsymbol{h}\left(\boldsymbol{q}^{1} \text {.data }, \ldots, \boldsymbol{q}^{k} \text {.data }\right)\right] \text { : }}$ \\
\hline \multirow{2}{*}{$\begin{aligned} \alpha_{p_{i}}^{\prime}(x) & =\alpha_{\text {new }_{i}}^{\prime}(x), \quad \alpha_{q_{i}}^{\prime}(x)=\alpha_{q_{i-}}^{\prime} \\
\beta_{f_{i}}^{\prime}(x, y) & =\left(\beta_{f_{i-1}}^{\prime}(x, y) \wedge \neg \alpha_{p_{i-1}}^{\prime}(x)\right)\end{aligned}$} \\
\hline \\
\hline$\vee\left(\alpha_{\text {new }_{i}}^{\prime}(y) \wedge \exists e x . \quad\left(\beta_{f_{i-1}}^{\prime}(x, e x) \wedge \alpha_{p_{i-1}}^{\prime}(e x)\right)\right)$ \\
\hline $\operatorname{active}_{i}(x)=\left(\operatorname{active}_{i-1}(x) \wedge \neg p_{i-1}(x)\right) \vee \alpha_{\text {new }_{i}}^{\prime}(x)$ \\
\hline error $_{i}=\exists x \cdot\left(\bigvee_{z \in\left\{p, q^{1}, \ldots, q^{k}\right\}}\left(\alpha_{z_{i-1}}^{\prime}(x) \wedge \alpha_{x n i i_{i-1}}^{\prime}(x)\right)\right)$ \\
\hline
\end{tabular}

Figure 2. Predicates defining the new data-structure.

adapt Pre to the trail for index 0 , which corresponds to the original graph, i.e. the predicates $p$ are replaced with $p_{0}$, for every $p \in V a r$, and the edge predicates $f$ with $f_{0}$, for every $f \in F$. Moreover, whenever we refer to a node in the graph we need to be sure that node is active which can be done by using the predicate active $0(x)$ which holds true if $x$ is in the first subtree of the root and $\psi_{U}^{\prime}(x)$ holds. A similar transformation is done for the formula $\neg$ Post, where now we consider pointers, edge labels, and active nodes at the last step $m$. Let $\operatorname{Pre}_{\mathcal{R}_{P}}$ (resp., Post $\mathcal{R}_{P}$ ) be the STRAND formula corresponding to the adaptation of Pre (resp., Post)

Reduction to satisfiability problem on the trail. It is easy to see that an error occurs during the execution of $P$ on a graph defined through $\mathcal{R}$ that satisfies Pre if the following STRAND formula is satisfiable on the trail $\mathcal{R}_{P}$ :

$$
\text { Error }=\bigvee_{i \in[m]}\left(\operatorname{Pre}_{\mathcal{R}_{P}} \wedge \bigwedge_{j \in[i-1]} \varphi_{j} \wedge \text { error }_{i}\right)
$$

Similarly, the Hoare-triple is not valid iff the following STRAND formula is satisfiable on the trail:

$$
\text { Violate }_{\text {Post }}=\operatorname{Pre}_{\mathcal{R}_{P}} \wedge\left(\bigwedge_{j \in[m]} \varphi_{j}\right) \wedge \neg \text { Post }_{\mathcal{R}_{P}}
$$

THEOREM 6.1. Let $P$ be a program, $\mathcal{R}$ be a recursive datastructure, and Pre, Post be two STRAND $\exists, \forall$ formulas over Var, $F$, and data. Then, there is a graph $G \in \operatorname{Graph}(\mathcal{R})$ that satisfies Pre and where either $P$ terminates with an error or the obtained graph $G^{\prime}$ does not satisfy Post iff the STRAND formula Error $\vee$ Violate $_{\text {Post }}$ is satisfiable on the trail $\mathcal{R}_{P}$.

\section{Evaluation}

\subsection{Implementation}

In this section, we demonstrate the effectiveness and practicality of the decision procedures for STRAND by checking verification conditions generated in proving properties of several heapmanipulating programs. Given pre-conditions, post-conditions and loop-invariants, each linear block of statements of a program yields a Hoare-triple, which is manually translated into a STRAND formula $\psi$ over trees and integer arithmetic, as a verification condition.

The decision procedure for STRAND implements the decision procedure for the semantically defined fragment STRAND dec $^{\text {sem }}$. Given a STRAND formula, our procedure will first determine if it is in the semantic decidable fragment, and if not, will halt and report that satisfiability of the formula is not checkable. When given a formula in the syntactic fragment $\mathrm{STRAND}_{d e c}$, this procedure will always succeed, and the decision procedure will determine satisfiability of the formula.

The decision procedure consists of a structural phase, where we determine whether the number of minimal models is finite, and if so, determine a bound on the size of the minimal models. This phase is effected by using MONA [13], a monadic second-order logic solver over (strings and) trees. In the second data-constraint solving phase, the finite set of minimal models, if any, are examined by the data-solver Z3 [9] to check if they can be extended with data-values to satisfy the formula.

Instead of building an automaton representing the minimal models and then checking it for finiteness, we check the finiteness formula MinModel $_{\psi}$ using WS2S, supported by MonA, which is a monadic second-order logic over infinite trees with setquantification restricted to finite sets. By quantifying over a finite universe $U$, and transforming all quantifications to be interpreted over $U$, we can interpret MinModel ${ }_{\psi}$ over all finite trees. Let us denote this emulation as MinModel ${ }_{U, \psi}^{\prime}$. The finiteness condition can now be checked by asking if there exists a finite set $B$ such that any minimal model for $\psi$ is contained within the nodes of $B$ :

$$
\exists \text { Bound } \forall U \quad \forall Q_{a_{(a \in \Sigma)}}\left(\text { MinModel }_{U, \psi}^{\prime} \Rightarrow(U \subseteq \text { Bound })\right)
$$

This formula has no free-variables, and hence either holds on the infinite tree or not, and can be checked by MONA. This formula evaluates to true iff the formula is in STRAND dec .

We also follow a slightly different procedure to synthesize the data-logic formula. Instead of extracting each minimal model, and 


\begin{tabular}{|c|c|c|c|c|c|c|c|c|c|c|}
\hline \multirow[b]{2}{*}{ Program } & \multirow[b]{2}{*}{$\begin{array}{l}\text { Verification } \\
\text { condition }\end{array}$} & \multicolumn{5}{|c|}{ Structural solving (MONA) } & \multicolumn{4}{|c|}{ Data-constraint Solving (Z3 with QF-LIA) } \\
\hline & & $\begin{array}{l}\text { in STRAND } \text { inc }_{\text {sem }} \\
\text { (finitely-many } \\
\text { minimal models) }\end{array}$ & \#States & $\begin{array}{r}\text { Final } \\
\text { BDD } \\
\text { size }\end{array}$ & Time(s) & $\begin{array}{l}\text { Graph } \\
\text { model } \\
\text { exists? }\end{array}$ & $\begin{array}{r}\text { Bound } \\
\text { (\#Nodes) }\end{array}$ & $\begin{array}{r}\text { Formula } \\
\text { size } \\
(\mathrm{KB})\end{array}$ & Satisfiable? & Time(s) \\
\hline \multirow{3}{*}{$\begin{array}{c}\text { sorted- } \\
\text { list-search }\end{array}$} & before-loop & Yes & 67 & 264 & 0.34 & No & - & - & - & - \\
\hline & in-loop & Yes & 131 & 585 & 0.59 & No & - & - & - & - \\
\hline & after-loop & Yes & 67 & 264 & 0.18 & No & - & - & - & - \\
\hline \multirow{4}{*}{$\begin{array}{l}\text { sorted- } \\
\text { list-insert }\end{array}$} & before-head & Yes & 73 & 298 & 1.66 & Yes & 5 & 6.2 & No & 0.02 \\
\hline & before-loop & Yes & 259 & 1290 & 0.38 & No & - & - & - & - \\
\hline & in-loop & Yes & 1027 & 6156 & 4.46 & No & - & - & - & - \\
\hline & after-loop & Yes & 146 & 680 & 13.93 & Yes & 7 & 14.5 & No & 0.02 \\
\hline $\begin{array}{l}\text { sorted-list- } \\
\text { insert-error }\end{array}$ & before-loop & Yes & 298 & 1519 & 0.34 & Yes & 7 & 9.5 & Yes & 0.02 \\
\hline \multirow{3}{*}{$\begin{array}{l}\text { sorted- } \\
\text { list-reverse }\end{array}$} & before-loop & Yes & 35 & 119 & 0.24 & No & - & - & - & - \\
\hline & in-loop & Yes & 513 & 2816 & 2.79 & No & - & - & - & - \\
\hline & after-loop & Yes & 129 & 576 & 0.35 & No & - & - & - & - \\
\hline \multirow{3}{*}{ bubblesort } & loop-if-if & Yes & 2049 & 13312 & 7.70 & No & - & - & - & - \\
\hline & loop-if-else & Yes & 1025 & 6144 & 6.83 & No & - & - & - & - \\
\hline & loop-else & Yes & 1033 & 6204 & 2.73 & Yes & 8 & 22.2 & No & 0.02 \\
\hline \multirow{3}{*}{ bst-search } & before-loop & Yes & 52 & 276 & 5.03 & No & - & - & - & 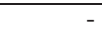 \\
\hline & in-loop & Yes & 160 & 1132 & 32.80 & Yes & 9 & 7.7 & No & 0.02 \\
\hline & after-loop & Yes & 52 & 276 & 3.27 & No & - & - & - & - \\
\hline \multirow{3}{*}{ bst-insert } & before-loop & Yes & 36 & 196 & 1.34 & No & - & - & - & - \\
\hline & in-loop & Yes & 68 & 452 & 9.84 & No & - & - & - & - \\
\hline & after-loop & Yes & 20 & 84 & 1.76 & No & - & - & - & - \\
\hline left/right-rotate & bst-preserving & Yes & 29 & 117 & 1.59 & Yes & 19 & 70.3 & No & 0.05 \\
\hline
\end{tabular}

Figure 3. Results of program verification

checking if there is a data-extension for it, we obtain a bound on the size of minimal models, and ask the data-solver to check for any model within that bound. This is often a much simpler formula to feed to the data-solver.

In our current implementation, the MoNA constraints are encoded manually, and once the bound is obtained, we write a program that outputs the $\mathrm{Z} 3$ constraints for the verification condition and the bound. The translation from STRAND to MONA formulas and the translation from STRAND formulas to Z3 formulas for any bound can be automated, and is a plan for the future.

\subsection{Experiments}

Figure 3 presents the evaluation of our tools on checking a set of programs that manipulate sorted singly-linked lists and binary search trees. Note that the binary search trees presented here are out of the scope of the logics HAVOC [16] and CSL [7].

The programs sorted-list-search and sorted-listinsert search and insert a node in a sorted singly-linked list, respectively, while sorted-list-insert-error is the insertion program with an intended error. The program sorted-list -reverse is a routine for in-place reversal of a sorted singlylinked list, which results in a reverse-sorted list, and bubblesort is the code for Bubble-sort of a list. The routines bst-search and bst-insert search and insert a node in a binary search tree, respectively, while the programs left-rotate and right-rotate perform rotations (for balancing) in a binary search tree.

For all these examples, a set of partial correctness properties including both structural and data requirements is checked. For example, assuming a node with value $k$ exists, we check if both sorted-list-search and bst-search return a node with value $k$. For sorted-list-insert, we assume that the inserted value does not exist, and check if the resulting list contains the inserted node, and the sortedness property continues to hold. In the program bst-insert, assuming the tree does not contain the inserted node in the beginning, we check whether the final tree contains the inserted node, and the binary-search-tree property continues to hold. In sorted-list-reverse, we check if the output list is a valid list that is reverse-sorted. The code for bubblesort is checked to see if it results in a sorted list. And the left-rotate and right-rotate codes are checked to see whether they maintain the property that maintain the binary search-tree property.

Note that each program requires checking several verification conditions (typically for the linear block from the beginning of the program to a loop, for the loop invariant linear block, and for the block from the loop invariant to the end of the program).

The experiments were conducted on a $2.2 \mathrm{GHz}, 4 \mathrm{~GB}$ machine running Windows 7 , and the formulas and results are available at http://www.cs.uiuc.edu/ qiu2/strand.

For the structural solving phase, we report first whether the verification condition falls within our semantic decidable fragment $\mathrm{STRAND}_{d e c}^{\text {sem }}$. In fact, it turns out that all of our verification conditions can be written entirely in the syntactic decidable fragment STRAND dec!

We also report the number of states, the BDD sizes to represent automata, and the time taken by MONA to compute the minimal models. We report whether there were any models found; note that if the formula is unsatisfiable and there are no models, the $\mathrm{Z} 3$ phase is skipped (these are denoted by “-” annotations in the table for Z3).

For the data-constraint solving phase, we first report the number of nodes of the tree (or string) that is an upper bound for all minimal models. The Z3 formulas are typically large (but simple) as one can see from the size of the formulas in the table. We report whether Z3 found the formula to be satisfiable or not (all cases were unsatisfiable, except sorted-list-insert-error, as the Hoaretriples verified were correct), and the time it took to determine this.

The experimental results show that natural verification conditions tend to be expressible in the syntactic decidable fragment $\mathrm{STRAND}_{d e c}$. Moreover, the expressiveness of our logic allows us to write complex conditions involving structure and data, and yet are handled well by MONA and Z3. We believe that a full-fledged engineering of an SMT solver for STRAND ${ }_{d e c}^{s e m}$ that answers queries involving heap structures and data is a promising future direction. Towards this end, an efficient non-automata theoretic decision procedure (unlike MONA) that uses search techniques (like SAT) instead of representing the class of all models (like BDDs and automata) may yield more efficient decision procedures. 


\section{Related Work}

We first discuss related work that can reason with combinations of heaps and data. In handling heaps, first-order theories that can reason with restricted forms of the reachability relation for ensuring decidability are the most common. The work most closely related to our work is the logic in HAVOC, called LISBQ [16], that offers a reasoning with generic heaps combined with an arbitrary datalogic. The logic has restricted reachability predicates and universal quantification, but is syntactically severely curtailed, to obtain decidability. We find the restrictions on the syntax quite awkward, with sort-based restrictions in the logic. Furthermore, the logic cannot handle even simple constraints over trees with unbounded depth where the nodes are of the same sort (like a tree being a binary search tree). However, the logic is extremely efficient, as it uses no structural solver, but translates the structure-solving also to (the Boolean aspect of) the SMT solver. We gained a lot of insight into decidability by studying the expressive power of HAVOC, and we believe that STRAND generalizes some of the underlying ideas present in HAVOC to a much more powerful technique for decidability. The logic CSL [7] has a similar flavor as HAVOC, with similar sort-restrictions on the syntax, but generalizes to handle doubly linked lists, and allows size constraints on structures. The work reported in [5] gives a logic that extends an LTL-like syntax to define certain decidable logic fragments on heaps.

Rakamarić et al [23] propose an inference rule system for reasoning with restricted reachability (but this logic does not have universal quantification and cannot express disjointness constraints), and an SMT solver based implementation has been reported [24]. Restricted forms of reachability were first axiomatized in early work by Nelson [21]. Several mechanisms without quantification exist, including the work reported in $[1,25]$. Automatic decision procedures that approximate higher-order logic using first-order logic, using approximate logics over sets and their cardinalities, have been proposed [15].

There is a rich literature on heap analysis without data. Since first-order logic over graphs is undecidable, decidable logics must either restrict the logic or the class of graphs. The closest work to ours in this realm is PALE [20], which restricts structures to be definable over tree-skeletons, similar to STRAND, and reduces problems to the Mona system [13]. Several approximations of firstorder axiomatizations of reachability have been proposed: axioms capturing local properties [19], a logic on regular patterns that is decidable [28], among others.

Finally, separation logic [26] has emerged as a convenient logic to express heap properties of programs, and a decidable fragment (without data) on lists is known [4]. However, not many extensions of separation logics handle data constraints (see [18] which combines this logic for linked lists with arithmetic).

\section{Acknowledgments}

We thank Christof Löding for the fruitful discussions we had when we started this project a few years back. This work is partially funded by NSF CAREER award \#0747041 and French ANR-09SEGI project Veridyc.

\section{References}

[1] I. Balaban, A. Pnueli, and L. D. Zuck. Shape analysis by predicate abstraction. In VMCAI'05, volume 3385 of $L N C S$, pages 164-180. Springer, 2005.

[2] T. Ball, R. Majumdar, T. Millstein, and S. K. Rajamani. Automatic predicate abstraction of C programs. In PLDI'01, pages 203-213. ACM, 2001.

[3] M. Barnett, B.-Y. E. Chang, R. DeLine, B. Jacobs, and K. R. M. Leino. Boogie: A modular reusable verifier for object-oriented programs. In FMCO'05, volume 4111 of LNCS, pages 364-387. Springer, 2005.
[4] J. Berdine, C. Calcagno, and P. W. O'Hearn. A decidable fragment of separation logic. In FSTTCS'04, volume 3328 of LNCS, pages $97-$ 109. Springer, 2004.

[5] N. Bjørner and J. Hendrix. Linear functional fixed-points. In $C A V$ '09, volume 5643 of $L N C S$, pages 124-139. Springer, 2009.

[6] E. Börger, E. Grädel, and Y. Gurevich. The Classical Decision Problem. Springer, 2001.

[7] A. Bouajjani, C. Dragoi, C. Enea, and M. Sighireanu. A logicbased framework for reasoning about composite data structures. In CONCUR'09, volume 5710 of LNCS, pages 178-195. Springer, 2009.

[8] L. M. de Moura and N. Bjørner. Deciding effectively propositional logic using DPLL and substitution sets. In IJCAR'08, volume 5195 of LNCS, pages 410-425. Springer, 2008.

[9] L. M. de Moura and N. Bjørner. Z3: An efficient SMT solver. In TACAS'08, volume 4963 of LNCS, pages 337-340. Springer, 2008.

[10] J. Engelfriet. Context-free graph grammars. In Handbook of Formal Languages, volume 3, pages 125-214. Springer, 1997.

[11] C. Flanagan, K. R. M. Leino, M. Lillibridge, G. Nelson, J. B. Saxe, and R. Stata. Extended static checking for Java. In PLDI'O2, pages 234-245. ACM, 2002.

[12] P. Godefroid, N. Klarlund, and K. Sen. DART: directed automated random testing. In PLDI'05, pages 213-223. ACM, 2005.

[13] N. Klarlund and A. Møller. MONA. BRICS, Department of Computer Science, Aarhus University, January 2001. Available from http://www.brics.dk/mona/.

[14] N. Klarlund and M. I. Schwartzbach. Graph types. In POPL'93, pages 196-205. ACM, 1993.

[15] V. Kuncak. Modular Data Structure Verification. PhD thesis, Massachusetts Institute of Technology, 2007.

[16] S. Lahiri and S. Qadeer. Back to the future: revisiting precise program verification using SMT solvers. In POPL'08, pages 171-182. ACM, 2008.

[17] T. Lev-Ami and S. Sagiv. Tvla: A system for implementing static analyses. In $S A S^{\prime} 00$, volume 1824 of $L N C S$, pages 280-301. Springer, 2000

[18] S. Magill, M.-H. Tsai, P. Lee, and Y.-K. Tsay. THOR: A tool for reasoning about shape and arithmetic. In $C A V^{\prime} 08$, volume 5123 of LNCS, pages 428-432. Springer, 2008.

[19] S. McPeak and G. C. Necula. Data structure specifications via local equality axioms. In $C A V^{\prime} 05$, volume 3576 of $L N C S$, pages 476-490. Springer, 2005

[20] A. Møller and M. I. Schwartzbach. The pointer assertion logic engine. In PLDI'01, pages 221-231. ACM, 2001.

[21] G. Nelson. Verifying reachability invariants of linked structures. In POPL'83, pages 38-47. ACM, 1983.

[22] G. Nelson and D. C. Oppen. Simplification by cooperating decision procedures. ACM Trans. Program. Lang. Syst., 1:245-257, 1979.

[23] Z. Rakamarić, J. D. Bingham, and A. J. Hu. An inference-rule-based decision procedure for verification of heap-manipulating programs with mutable data and cyclic data structures. In VMCAI'07, volume 4349 of LNCS, pages 106-121. Springer, 2007.

[24] Z. Rakamarić, R. Bruttomesso, A. J. Hu, and A. Cimatti. Verifying heap-manipulating programs in an SMT framework. In ATVA'07, volume 4762 of LNCS, pages 237-252. Springer, 2007.

[25] S. Ranise and C. Zarba. A theory of singly-linked lists and its extensible decision procedure. In SEFM'06, pages 206-215. IEEE-CS, 2006.

[26] J. Reynolds. Separation logic: a logic for shared mutable data structures. In LICS'02, pages 55-74. IEEE-CS, 2002.

[27] G. Yorsh, T. W. Reps, and S. Sagiv. Symbolically computing mostprecise abstract operations for shape analysis. In TACAS'04, volume 2988 of $L N C S$, pages 530-545. Springer, 2004.

[28] G. Yorsh, A. M. Rabinovich, M. Sagiv, A. Meyer, and A. Bouajjani. A logic of reachable patterns in linked data-structures. In FoSSaCS'06, volume 3921 of $L N C S$, pages 94-110. Springer, 2006. 